ARTICLE

\title{
Gating mechanism of elongating $\beta$-ketoacyl-ACP synthases
}

Jeffrey T. Mindrebo 1,2,5, Ashay Patel 1,5, Woojoo E. Kim¹, Tony D. Davis (1) 1, Aochiu Chen (1) 1, Thomas G. Bartholow1, James J. La Clair ${ }^{1,2}$, J. Andrew McCammon 1,3, Joseph P. Noel (D) ${ }^{1,2,4 凶}$ \& Michael D. Burkart (1D ${ }^{1 凶}$

Carbon-carbon bond forming reactions are essential transformations in natural product biosynthesis. During de novo fatty acid and polyketide biosynthesis, $\beta$-ketoacyl-acyl carrier protein (ACP) synthases (KS), catalyze this process via a decarboxylative Claisen-like condensation reaction. KSs must recognize multiple chemically distinct ACPs and choreograph a ping-pong mechanism, often in an iterative fashion. Here, we report crystal structures of substrate mimetic bearing ACPs in complex with the elongating KSs from Escherichia coli, FabF and FabB, in order to better understand the stereochemical features governing substrate discrimination by KSs. Complemented by molecular dynamics (MD) simulations and mutagenesis studies, these structures reveal conformational states accessed during KS catalysis. These data taken together support a gating mechanism that regulates acyl-ACP binding and substrate delivery to the KS active site. Two active site loops undergo large conformational excursions during this dynamic gating mechanism and are likely evolutionarily conserved features in elongating KSs.

\footnotetext{
${ }^{1}$ Department of Chemistry and Biochemistry, University of California, San Diego, 9500 Gilman Drive, La Jolla, CA 92093-0358, USA. ${ }^{2}$ Jack H. Skirball Center for Chemical Biology and Proteomics, Salk Institute for Biological Studies, La Jolla, CA 92037, USA. ${ }^{3}$ Department of Pharmacology, University of California, San Diego, 9500 Gilman Drive, La Jolla, CA 92093, USA. ${ }^{4}$ Howard Hughes Medical Institute, Salk Institute for Biological Studies, La Jolla, CA 92037, USA.

${ }^{5}$ These authors contributed equally: Jeffrey T. Mindrebo, Ashay Patel ${ }^{\circledR}$ email: noel@salk.edu; mburkart@ucsd.edu
} 
F atty acid synthases (FASs) and polyketide synthases (PKSs) iteratively condense and modify ketide units to produce a variety of natural compounds, ranging from fatty acids to complex bioactive molecules ${ }^{1,2}$. FASs and PKSs can exist as one or more polypeptide mega-synthases that contain distinct catalytic domains (type I) or as discrete enzymes, each possessing specific activities (type II) ${ }^{3-8}$. In these pathways, $\beta$-ketoacyl ACP synthases, alternatively ketosynthases (KSs), catalyze carbon-carbon formation via a precisely choreographed decarboxylative Claisen-like condensation to produce a $\beta$-ketoacyl species. In fatty acid biosynthesis (FAB, Fig. 1a), complete reduction at the $\beta$-position occurs before further chain extension, while in polyketide biosynthesis (PKB), the $\beta$-positions of pathway intermediates can be completely, partially, or not reduced to produce structurally and functionally complex natural products ${ }^{1,9}$. The small, $4-\alpha$-helical acyl carrier protein (ACP) is used to shuttle substrates and intermediates to each catalytic domain or discrete enzyme in these pathways ${ }^{10,11}$. ACPs are initially translated to an inactive apo form and are subsequently post-translationally modified to an active holo form via attachment of a 4'-phosphopantetheine (PPant) arm at a conserved serine residue, providing a thiol moiety that ligates substrates and pathway intermediates to the $\mathrm{ACP}^{10}$. Protein-protein interactions (PPIs) between ACPs and their enzymatic partners (or domains) regulate the catalytic activities of FASs and PKSs and additionally provide a mechanism to ensure pathway orthogonality between primary (i.e., FAB) and secondary metabolism (i.e., $\mathrm{PKB})^{3,5,6,12-17}$.

KS-catalyzed carbon-carbon bond formation is an essential reaction common to both $\mathrm{FAB}$ and $\mathrm{PKB}$. The decarboxylative condensation reaction provides a thermodynamic driving force for the formation of complex natural products ${ }^{18,19}$. In addition, KS domains play an important role in chain length determination, ensuring product fidelity, and controlling pathway flux ${ }^{15,20,21}$. Therefore, efforts to engineer FASs and PKSs will depend on our ability to manipulate the specificities and activities of these domains/enzymes, as evidenced by the recent successes engineering the yeast $\mathrm{FAS}^{21-26}$

KSs can be classified as elongating or initiating based on their role within a given pathway. Initiating KSs serve to catalyze the first condensation reaction of $\mathrm{FAB}$ or PKB pathways, converting holo-ACP to acyl-ACP, while all subsequent condensation reactions are catalyzed by elongating $\mathrm{KSs}^{3,4}$. Despite their distinct biosynthetic roles, both types of KS operate via a common ping-pong reaction mechanism, which can be disaggregated into two discrete steps $s^{21,27,28}$. In the first step, an acyl-ACP associates with a KS, and its acyl cargo is transferred from the PPant arm to a conserved, active site cysteine, producing a covalent acyl-KS adduct and holo-ACP. In the second step, malonyl-ACP replaces holo-ACP as the KS's binding partner, subsequently undergoing a decarboxylative Claisen-like condensation with the acyl-KS adduct that produces $\beta$-ketoacyl-ACP (Supplementary Fig. 1). During the course of this ping-pong process, two distinct ACPs must sequentially interact with the KS, and thus must enforce exquisite temporal and spatial discrimination among the subtly different chemical states of these acyl-ACPs. The mechanisms used by KSs to coordinate these two half-reactions and how this coordination determines substrate specificity remains cryptic despite extensive biochemical and structural characterization.

The E. coli type II FAS has served as a model system for understanding ACP-mediated PPIs and FAB ${ }^{19,29-36}$. Here, we use this well-characterized system to better understand ACP.KS PPIs and KS substrate discrimination by structurally characterizing $E$. coli elongating KSs, FabB (KASI family KS), and FabF (KASII a

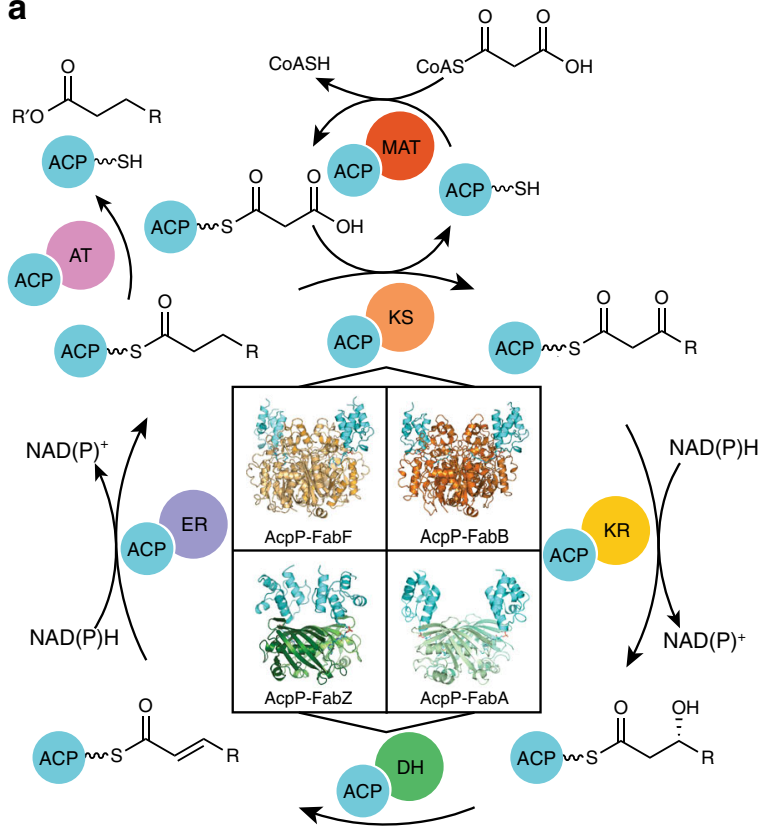

b

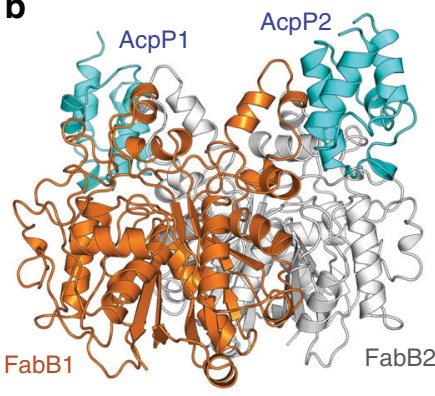

C

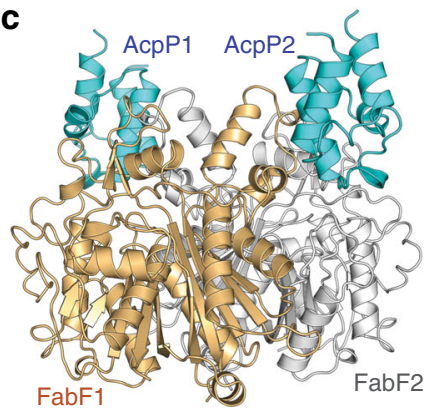

d

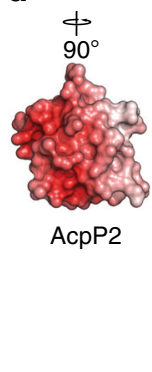

e

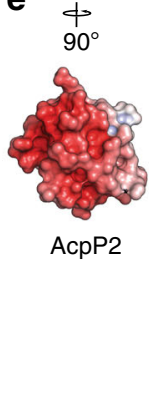

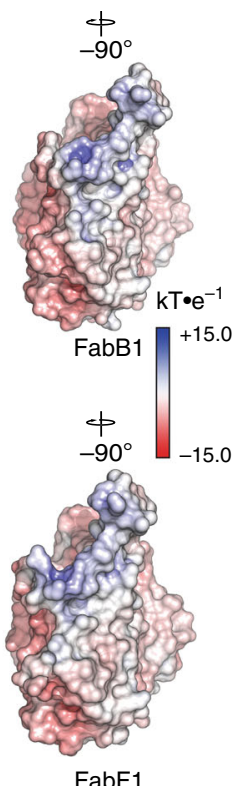

FabF1

Fig. 1 FAB cycle and known AcpP-PP complexes. a A schematic representation of the chemical transformations that occur during fatty acid biosynthesis, highlighting the importance of protein-protein interactions required at each step for substrate processing. The four quadrants at the center of the scheme show the structures of all FAS AcpP crosslinked complexes solved thus far. The top two structures are the crosslinked AcpP-KSs (AcpP-FabF and AcpP-FabB) reported herein, while depicted below are the crosslinked AcpP-DHs (AcpP-FabZ and AcpP-FabA) solved and reported in previous publications ${ }^{14,33}$. Each domain is abbreviated as follows: MAT malonyl-ACP transacylase, KS ketosynthase, KR ketoreductase, DH dehydratase, ER enoylreductase, and AT acyl transferase. AcpP-DH structures depicted in bottom two quadrants are AcpP-FabZ (PDB: 6N3P), AcpP-FabA (PDB: 4KEH). b, c Cartoon representations of the crosslinked AcpP-FabB and AcpP-FabF dimers. AcpP is colored cyan; the first KS monomer of AcpP-FabB and AcpPFabF is colored orange or light orange, respectively, while the second is colored white in both structures. $\mathbf{d}$ Electrostatic potentials (ESP) maps of the FabB and AcpP monomers. e ESP maps of the FabF and AcpP monomers. In all cases, the ESP is mapped onto the Connelly surfaces of the FabF and AcpP monomers using a blue to white to red color range, spanning from $+15.0 \mathrm{kT} \cdot \mathrm{e}^{-1}$ to $-15.0 \mathrm{kT} \cdot \mathrm{e}^{-1}$. 
a<smiles>CC(Br)C(=O)NCCNC(=O)CCNC(=O)[C@@H](O)C(C)(C)CO</smiles><smiles>CC(C)(CO)[C@H](O)C(=O)NCCC(=O)NCCNC(=O)C=C(P)Cl</smiles>

$2 \mathbf{2 a}: \mathrm{R}=\mathrm{H}$
$2 \mathbf{b}: \mathrm{R}=\mathrm{C}_{5} \mathrm{H}_{11}$

b

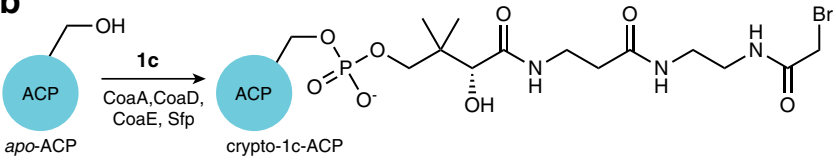

Fig. 2 KS pantetheineamide crosslinking probes and ACP one-pot

method. a Pantetheineamide-derived $\alpha$-bromo and chlorovinyl crosslinking probes used in this study. $\mathbf{b}$ An illustration of the one-pot chemoenzymatic loading of apo-ACP with panetheinamide probe $\mathbf{1 c}$ to produce crypto-1c$\mathrm{ACP}$ using the coenzyme $\mathrm{A}$ biosynthetic enzymes CoaA, CoaD, and CoaE and a promiscuous phosphopantetheinyl transferase (PPTase) Sfp.

family KS), as substrate/intermediate complexes with ACP that approximate states formed during catalysis. In conjunction with molecular dynamics (MD) simulations and mutagenesis studies, these structures provide dynamical descriptions of two ACP.KS complexes, revealing conformational changes that regulate substrate processing. Specifically, we propose that the elongating KSs employ two conserved active site loops that open and close through a double drawbridge-like gating mechanism in order to regulate substrate processing ${ }^{37}$. When open, the drawbridge expands the KS active site in order to accommodate PPanttethered acyl-AcpP substrates. The structural features underlying this gating machinery are conserved in related enzymes and similar conformational transitions will likely determine substrate selectivity and processing in other FAB and PKB pathways.

\section{Results}

Crosslinking and crystallization of AcpP-KS complexes. We synthesized C12-a-bromo- (1a) and C16- $\alpha$-bromopantetheineamide (1b) and loaded them onto the E. coli ACP (AcpP) using a one-pot chemoenzymatic method ${ }^{38}$ to produce crypto-1a-AcpP (C12AcpP) and crypto-1b-AcpP (C16AcpP) that bear a reactive warhead on their respective PPant arms ${ }^{38-40}$ (Fig. 2). These cryptoAcpPs were then reacted with FabF and FabB to produce uniformly crosslinked complexes, referred to herein as AcpP-KS, where "-" denotes a crosslinked complex (Supplementary Figs. 1 and 2). Using this method, we grew crystals of C12AcpP-FabF and C16AcpPFabF and obtained x-ray crystallographic data sets with $2.35 \AA$ and $2.30 \AA$ resolution, respectively. The crystallographic asymmetric units (ASU) of these complexes were identical and contained one monomer of FabF crosslinked to a single AcpP. In addition, C12AcpP-FabB crystals diffracted to $1.55 \AA$ and C16AcpP-FabB diffracted to $2.50 \AA$. The $\mathrm{C} 12 \mathrm{AcpP}-\mathrm{FabB}$ and $\mathrm{C} 16 \mathrm{AcpP}-\mathrm{FabB}$ ASUs both contain the functional FabB dimer crosslinked to two AcpP molecules (Fig. 2b, c). Additional analysis for the C12AcpP-FabF and C16AcpP-FabB structures can be found in Supplementary Notes 1 and 2.

The AcpP-KS interface. The AcpP-KS interacting surfaces are electrostatic (Fig. 2b-e) and composed largely of flanking charged and polar groups surrounding a central hydrophobic patch (Fig. 3). The AcpP and KS monomers provide negatively and positively charged residues, respectively, to the interface. Figure 3 provides a comparison of the AcpP-FabF and AcpP-FabB interfaces from this work. While a thorough analysis of the AcpP-FabB interface was recently performed by Milligan et al. ${ }^{34}$, herein we report an example of a KASII-type KS in complex with an ACP, AcpP-FabF. The AcpP-FabF and AcpP-FabB complexes can be broken into three regions. Region 1 electrostatic interactions include Lys65, Arg68, and Lys69 of FabF interacting with Glu13, Gln14, Asp35, and Asp38 of AcpP (Fig. 3a). In addition, Thr270 of FabF, which sits on a flexible loop that undergoes conformational rearrangements upon acyl-AcpP binding, interacts with Asp35 of AcpP. Region 2 interactions are formed between Arg127, Lys128, and Arg206 of FabF and Glu47, Glu48, Glu53, and Asp56 of AcpP (Fig. 3b). The central hydrophobic patch is comprised of interactions between Met44, Val40, and Leu37 of AcpP and Ile129, Ser130, Pro131, Phe132, Ala205, and Arg206 of FabF (Fig. 3c).

Comparisons of the AcpP-FabF and AcpP-FabB interfaces reveal that they share similar chemical features, but structural alignments of the KS subunits show that the orientations of AcpPs in the AcpP-KS complexes are distinct (Supplementary Fig. 3a, b). In FabF, individual ACP monomers, AcpP1 and AcpP2, bury surface areas of $671.2 \AA^{2}$ and $675.4 \AA^{2}$, respectively, while in FabB, AcpP1, and AcpP2 bury surface areas of $597.5 \AA^{2}$ and $607.4 \AA^{2}$, respectively. These small contact areas are consistent with the transient nature of molecular recognition between ACP and its binding partners ${ }^{14,33}$. In addition, the differences in contact areas of AcpP-FabF and AcpP-FabB interfaces are consistent with the differing orientations that the AcpPs adopt relative to their KS partners. It is also worth noting that when comparing the interfaces of these AcpP-KS complexes, AcpP's helix I forms more extensive contacts with FabF than FabB, while FabB forms more hydrophobic interactions at region 3 (Fig. 3). While the differences at these interfaces are subtle, the manner in which AcpP engages with each of its cognate KSs may modulate the activities of FabF and $\mathrm{FabB}^{41,42}$. A timeresolved analysis of the FabF and FabB interfaces using $\mathrm{MD}$ simulation data is provided in Supplementary Note 3.

Changes in the FabF active site accompany substrate binding. Electron density maps calculated using the $2.30 \AA$ C16AcpP-FabF data set demonstrate adequate density for ACP's Ser36, the PPant moiety, and acyl cargo. The FabF active site cysteine residue (Cys163) forms a covalent bond with the a-carbon of the fatty acid substrate mimetic (1b), and the resulting stereocenter is found in the $S$ configuration (Fig. 4a). The carbonyl group of the substrate coordinates to the FabF active site histidine residues, His303 and His340, in a manner similar to the published structure of AcpP-FabB ${ }^{34}$ (PDB: 5KOF) (Fig. 4b). Modeling and refinement of the crosslinker's acyl chain required the remodeling of two active site loops. The first of which is the conserved GFGG loop (loop 1, residues 399-402) adjacent to the active site that contains the putative Phe400 gating residue ${ }^{27}$, and a second less conserved loop (loop 2, residues 265-275) that caps loop 1 (Fig. 5a). These loops undergo significant conformational changes, revealing a hydrophobic pocket that accommodates the acyl chain (Supplementary Fig. 4a-c and Supplementary Note 4). This pocket is distinct from the substrate binding site identified in the crystallized acyl-KS intermediate structures $2 \mathrm{GFY}^{43}$ and $1 \mathrm{EK} 4^{44}$ (Fig. 4c). Close analysis of the C16AcpP-FabF structure indicates that crystal contacts do not play a role in the conformational changes seen in these loops.

C16AcpP-FabF adopts an open conformation. A comparison of the apo-FabF (PDB: 2GFW) ${ }^{43}$, acyl-FabF (PDB: 2GFY) ${ }^{43}$, 

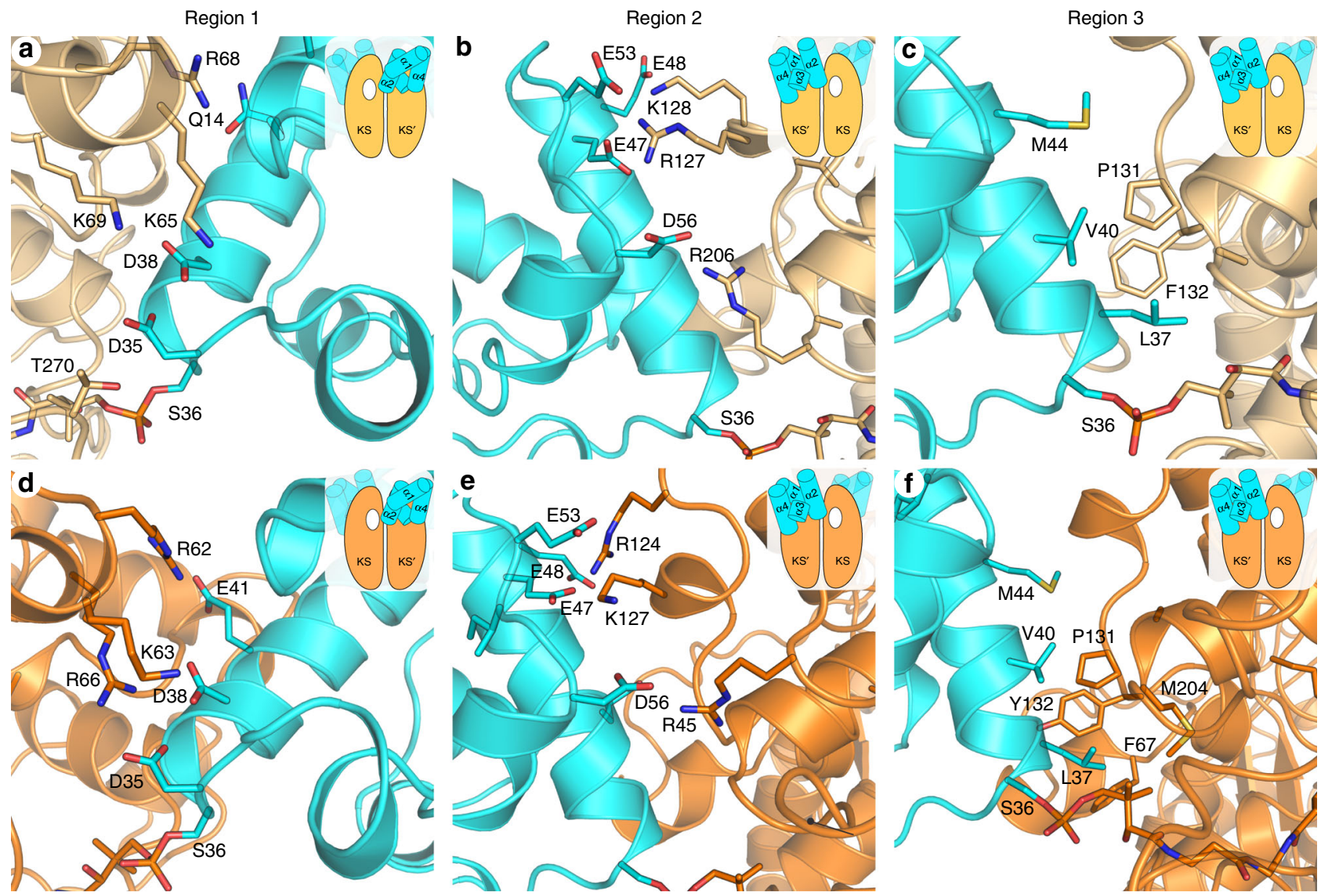

Fig. 3 AcpP-FabF and AcpP-FabB interface interactions. The FabF (light orange) and FabB (bright orange) interfaces can be broken up into three regions, the first two are comprised of electrostatic interactions and the third is a hydrophobic patch. The above panels provide close-up views of these three regions and the cartoon inset provides context regarding the viewing angle and the positional relationship of AcpP (cyan) to the overall AcpP-KS complex. In addition, the white hole represents the active site to which the viewed AcpP is crosslinked as the majority of contacts are provided by the KS monomer not covalently crosslinked to AcpP. a A close-up view of the AcpP-FabF region 1 electrostatic interactions. $\mathbf{b}$ A close-up view of the AcpP-FabF region 2 electrostatic interactions. c A close-up view of the AcpP-FabF region 3 hydrophobic interactions. d A close-up view of the AcpP-FabB region 1 electrostatic interactions. e A close-up view of the AcpP-FabB region 2 electrostatic interactions. f A close-up view of the AcpP-FabB region 3 hydrophobic interactions.

and C16AcpP-FabF structures shows that Phe400 of loop 1 moves $\sim 7 \AA$, as measured by changes in the Phe $400 \mathrm{Ca}$ position, into a pocket revealed by a conformational rearrangement of loop 2 (Fig. 5a). Gly399 and Gly402 of loop 1 act as pivot points as the loop transitions from a closed conformation to an open conformation (Fig. 5b, c). The open conformation is stabilized by a network of hydrogen-bonding interactions between the backbone amides of Gly399, Phe400, Gly401, and Gly402 and the side chains of Asn404 and Asp265 from loop 2 (Fig. 5b, c). Overlays of the $a p o-F a b F$ and $\mathrm{C} 16 \mathrm{AcpP}-\mathrm{FabF}$ structures indicate that the C16-acyl chain of $\mathbf{1 b}$ would clash with Phe400 in the closed conformation of loop 1 (Fig. 5a). These results indicate that these loop rearrangements may act as a gate to provide access to the active site for acyl-ACP substrates. Interestingly, the backbone amide of Phe 400 provides one of the two hydrogen bonds that form the FabF oxyanion hole when loop 1 assumes the closed conformation; therefore, the open conformation is likely catalytically inactive.

In apo-FabF, loop 2 rests on top of loop 1 and must undergo a rearrangement to allow loop 1 to assume the open conformation. A comparison of $a p o-F a b F$ to our C16AcpP-FabF structure demonstrates that loop 2 pivots about a Pro-Pro motif, whereby Pro273 undergoes a $165^{\circ}$ change in its $\psi$ angle, causing the loop to swing outwards by $5 \AA$ towards the bound AcpP (Fig. 5d). Analysis of the loop 2 backbone dihedral angles suggests that the loop transitions from a type VIII $\beta$-turn in the closed conformation to a type I $\beta$-turn in the open conformation. In the closed conformation, loop 2 is stabilized by a Ser271 ${ }_{(\mathrm{O}) \mathrm{C}}-\mathrm{His} 268_{(\mathrm{H}) \mathrm{N}}$ main-chain hydrogen bond, a Thr270His268 side-chain hydrogen bond, and a Pro273-Tyr267 C-H $\pi$ interaction $^{45}$ (Fig. 5e, f). Transition to the open conformation causes the $\beta$-turn backbone hydrogen-bonding pattern to flip from Ser271 $1_{(\mathrm{O}) \mathrm{C}^{-}} \mathrm{His} 268_{(\mathrm{H}) \mathrm{N}}$ to Ser271 ${ }_{(\mathrm{N}) \mathrm{H}^{-H}} \mathrm{His} 268_{(\mathrm{O}) \mathrm{C}}$ and Thr270 breaks its hydrogen bond with His268 to interact with Asp35 of AcpP.

C12AcpP-FabB trapped in closed acyl transfer conformation. Analysis of the $1.55 \AA$ C12AcpP-FabB data (Supplementary Fig. 4d) demonstrates well-resolved electron density for the $4^{\prime}$ phosphopantetheineamide arm and the C12-acyl chain. In this structure, loops 1 and 2 are found in a catalytically competent closed conformation (Fig. 4d-f). Like the catalytic cysteine of FabF, Cys163 of FabB, reacts stereoselectively with the a carbon of 1a to produce the $S$ stereoisomer (Fig. $4 \mathrm{~d}$ ). The carbonyl group of the substrate is coordinated to the oxyanion hole formed by the backbone amides of Cys163 and Phe400, and the probe's acyl chain is positioned inside FabB's fatty acid binding pocket (Supplementary Fig. 4d, e). Interestingly, the recently deposited AcpP-FabB structure (PDB: $5 \mathrm{KOF})^{34}$ does not feature these interactions, and instead the carbonyl group forms hydrogen bonds with His298 and His333. 

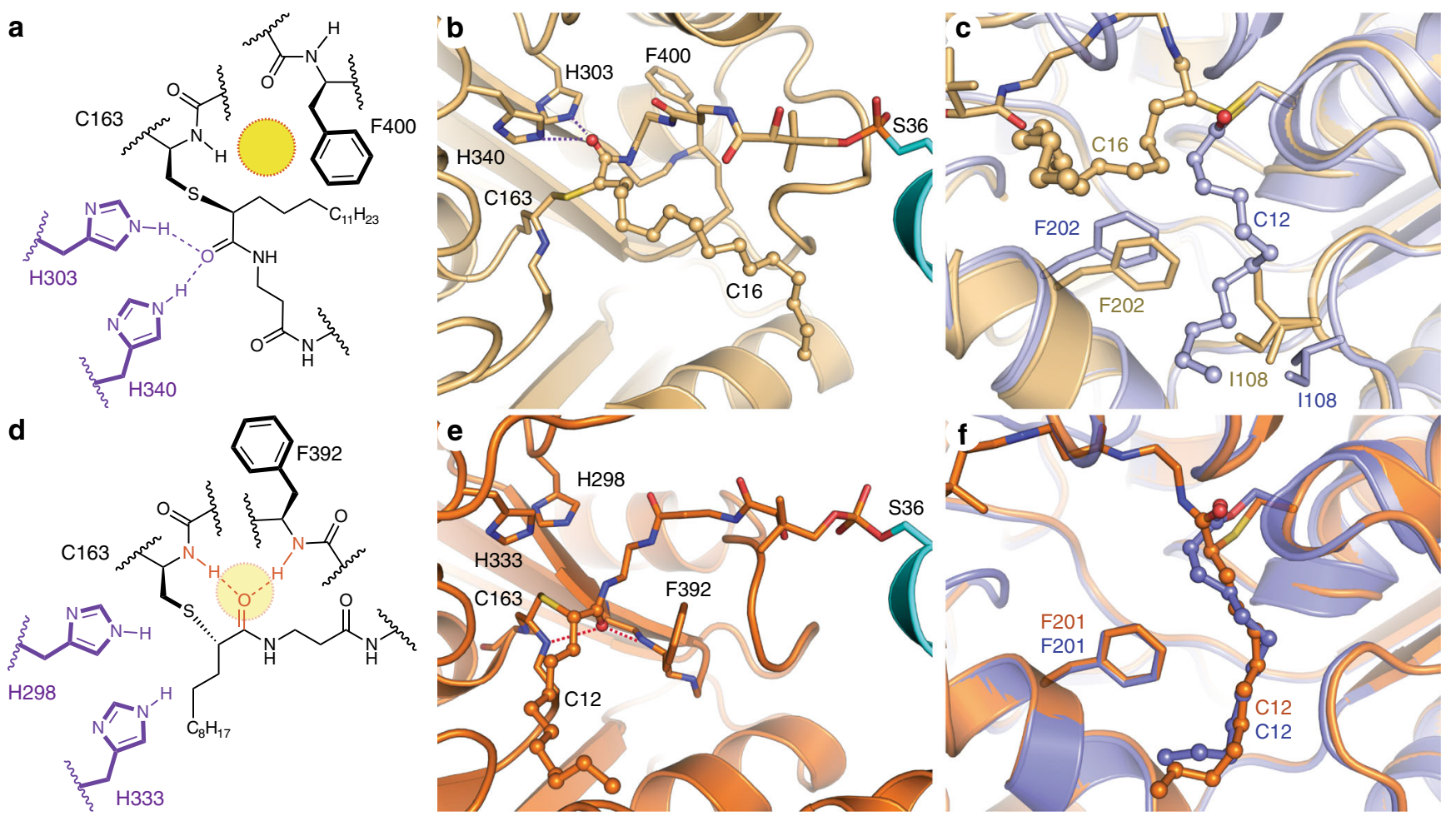

Fig. 4 FabF active site interactions with C16 substrate analog. a Schematic representation of the 4'-phosphopantetheineamide crosslinker within the FabF active site. The disorganized oxyanion hole is shown as a yellow circle. b Active site interactions depicting the PPant crosslinker and C16 acyl chain from the crystal structure of C16AcpP-FabF. H340 and $\mathrm{H} 303$ coordinate the carbonyl of the acyl mimetic (blue dotted lines) and the active site oxyanion hole is disorganized. AcpP and FabF are colored cyan and light orange, respectively. c Comparison of acyl binding pocket and associated gating residues, F202 and 1108, in dodecanoyl-FabF (12:0-FabF) (2GFY43, light purple), and C16AcpP-FabF (6OKG, light orange). d Schematic representation of the 4'-

phosphopantetheine crosslinker within the FabB active site. The organized oxyanion hole is shown as a transparent yellow circle with associated backbone amides and coordinated PPant carbonyl shown in red. e The carbonyl of the acyl mimetic in the crystal structure of C12AcpP-FabB is coordinated in the active site oxyanion hole formed by backbone amides of Cys163 and Phe392 (Phe400 in FabF). $f$ Comparison of acyl binding pocket in dodecanoyl-FabB (12:0-FabB) (PDB ID: 1EK444, bright purple), and C12AcpP-FabB (PDB ID: 6OKC, bright orange). Residues in panels are numbered according to their respective protein, FabF is numbered in FabF residue numbering and FabB is numbered in FabB residue numbering. Hydrogen-bonding interactions are represented as dotted lines, all side chain residues are represented as sticks, and the 4'-phosphopantetheinyl arm is represented as sticks while the acyl chains are represented as ball and stick.

Comparison of C12AcpP-FabB and dodecanoyl-FabB (C12FabB, PDB: 1EK4 $)^{44}$ demonstrates that the active site and acyl chain geometries are nearly identical in the two structures (Fig. 4f). The similarity between the C12-FabB and C12AcpP-FabB indicates that the latter approximates a catalytically relevant state for the transfer of substrate from acyl-AcpP to FabB's active site cysteine. Both steps of the KS reaction form a tetrahedral intermediate that is stabilized by the oxyanion hole, which is only organized when loop 1 and the associated Phe392 residue (Phe400 in $\mathrm{FabF}$ ), are in the gate-closed conformation.

MSA analysis suggests conservation of loops 1 and 2. Multiple sequence alignments (MSA) of type II FAS elongating KSs indicate that residues of loop 1 are highly conserved across the KASI (FabB) and KASII (FabF) families of KSs, while loop 2 is only conserved within, but not between, the two families (Supplementary Fig. 5). The consensus sequence for loop 1 is comprised of the conserved GFGG $\beta$-turn motif flanked by two conserved asparagine residues, Asn396 and Asn404 (FabF numbering). Interestingly, Asn404 interacts with a highly conserved Asp265 found at the start of loop 2 in both the open and closed states, providing a physical basis for the (proposed) coordinated movement of loops 1 and 2 (Fig. 5b, c). The conserved nature of loop 1 and Asp265 suggests that their proposed gating function is a general feature of type II FAS elongating KSs. Further analysis and comparison of FabF and FabB structure and function are provided in Supplementary Note 5.

To determine if these proposed gating elements are conserved in condensing enzymes outside of type II FAS, we aligned E. coli FabF with representative KSs from type II PKS, type I FAS, and type I PKS (Fig. 6a). Loop 1 of FAS and PKS KSs is generally conserved, although there is modest sequence variation. Notably, the Phe400 FabF gating residue is conserved in type I FAS and type II PKS but is replaced by valine or isoleucine in the KS domains of the type I PKS deoxyerythronolide B synthase (DEBS). Modification of the gating residue may modulate substrate specificity, as the KS domains from DEBS accept and extend substrates with $\beta$-substituents and utilize bulkier alphabranched extender units, namely methylmalonyl-CoA ${ }^{46}$.

Loop 2 of these KSs is significantly less conserved than loop 1. Only Asp265 can be considered highly conserved, as it is present in all KSs except the KS monomer (actKS) of the heterodimeric type II PKS KS/chain length factor (CLF) complex from actinorhodin biosynthesis ${ }^{47,48}$. In contrast to FAS KSs, these enzymes iteratively extend a growing polyketide within the KS binding pocket only unbinding the polyketide once multiple extensions using malonyl-ACP have been catalyzed to produce a fully extended poly- $\beta$-keto-ACP. The size of the polyketide produced by actKS is determined by CLF, which accommodates 

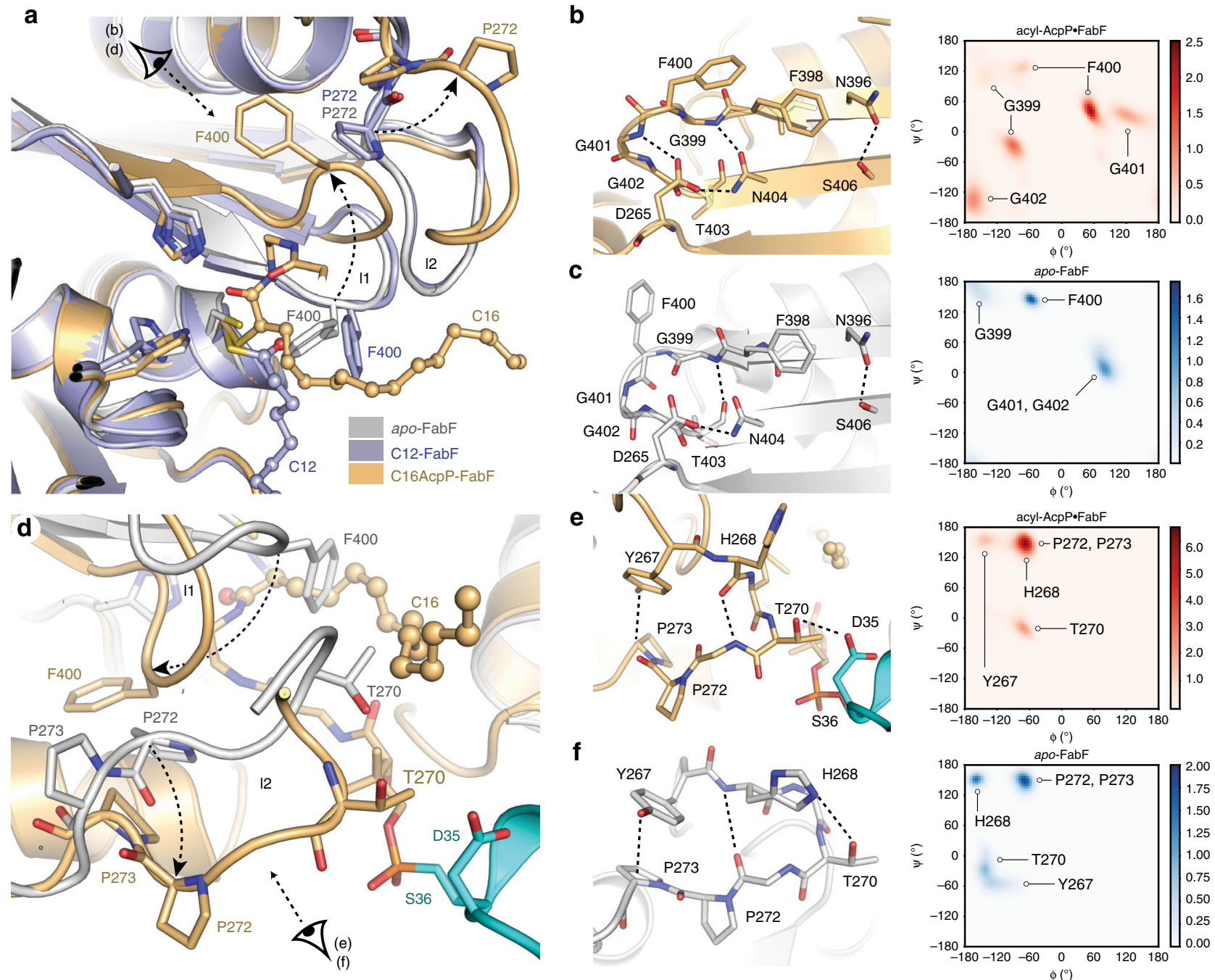

Fig. 5 Conformational changes in FabF active site loops. a Comparison of F400, loop 1, and loop 2 conformations of apo-FabF (light grey, PDB: 2GFW43), dodecanoyl-FabF (light purple, PDB: 2GFY43), and C16AcpP-FabF (light orange, PDB: 6OKG). b Interactions that stabilize the open-conformation of the $\beta$ turn motif of loop 1 in C16AcpP-FabF and associated Ramachandran analysis of the conserved GFGG sequence within loop 1 from 1.5 $\mu$ s of 10:0-AcpP.FabF, 12:0-AcpP.FabF, 16:0-AcpP.FabF (total of $4.5 \mu \mathrm{s}$ ) molecular dynamics (MD) simulation data. $\mathbf{c}$ Interactions that stabilize the closed-conformation of the $\beta$ turn motif of loop 1 in apo-FabF (light grey) and associated Ramachandran analysis of the conserved GFGG sequence within loop 1 from $1.5 \mu$ s of MD simulation data of apo-FabF. d Loop 2 overlay of apo-FabF (light grey, PDB: 2GFW) and C16AcpP-FabF (light orange) with the crosslinked AcpP colored cyan. e Interactions that stabilize the $\beta$-turn open conformation of loop 2 in C16AcpP-FabF (light orange) and associated Ramachandran analysis of key loop 2 residues of FabF monomers of acyl-AcpP.FabF complexes (gate-open conformation) from $1.5 \mu$ s of 10:0-AcpP.FabF, 12:0-AcpP.FabF, 16:0-AcpP.FabF (total of $4.5 \mu \mathrm{s}$ ) MD simulation data. $\mathbf{f}$ Interactions that stabilize the loop 2 closed conformation in apo-FabF (white) and associated Ramachandran analysis of key loop 2 residues of FabF of apo-FabF (gate-closed conformation) from $1.5 \mu$ s of MD simulation data of apo-FabF. a-f Residues are shown as sticks and colored according to element. Hydrogen-bond interactions are highlighted using dotted lines. $\mathbf{b}, \mathbf{c}, \mathbf{e}, \mathbf{f}$ Simulated backbone $\varphi$ and $\psi$ dihedrals were binned using widths of $5^{\circ}$. Color bars indicates density of data points within each $2 \mathrm{D}$ bin.

the growing polyketide chain, mediating substrate dissociation when the polyketide intermediate can no longer be accommodated within its binding pocket ${ }^{49}$. Therefore, given that iterative type II KSs do not accept acyl-ACPs and instead only process malonyl-ACPs, these iterative type II KSs may need to disfavor gating events to prevent premature product off-loading and ensure the growing polyketide is transferred back to the KS cysteine after condensation with malonyl-ACP. In actKS, this may be realized by disfavoring the open conformation of loops 1 and 2, relative to type II FAS KSs, through the substitution of the negatively charged Asp265 residue of loop 2 with an Asn residue (Fig. 6a), as the ion-dipole interactions involving Asp265 found in FabB or FabF are substituted with weaker dipole-dipole interactions in actKS. Interestingly, in KSs from non-iterative type II polyene PKSs, such as Iga11 $1^{50,51}$ and $\mathrm{ApeO}^{52}$, that require substrate offloading in-between condensation steps ${ }^{52}$, the same substitution is not observed, instead these enzymes possess the conserved Asp265 found in type II FAS KSs.

Mutagenesis studies of FabF gating mutants. To determine if the proposed gating function of loops 1 and 2 have any effect on KS activity, we identified a panel of FabF mutations that we hypothesized would attenuate FabF's ability to accept and/or process acyl-AcpP substrates. These mutations were designed to alter FabF's proposed gating function by modulating loop 1's flexibility (flex modulation), destabilizing (disfavoring) the gateopen conformation (destabilization), or occluding the pocket occupied by Phe400 in the gate-open conformation (pocket blockage). To test this hypothesis, we adapted a crosslinking assay 
a

\begin{tabular}{rccccc|c|c|cccc}
\multicolumn{110}{c|}{ Loop 1} \\
E. coli FabF & $\mathrm{C}$ & $\mathrm{N}$ & $\mathrm{S}$ & $\mathrm{F}$ & $\mathrm{G}$ & $\mathrm{F}$ & $\mathrm{G}$ & $\mathrm{G}$ & $\mathrm{T}$ & $\mathrm{N}$ & $\mathrm{G}$ \\
Iga11-KS & $\mathrm{N}$ & $\mathrm{N}$ & $\mathrm{S}$ & $\mathrm{S}$ & $\mathrm{A}$ & $\mathrm{F}$ & $\mathrm{A}$ & $\mathrm{G}$ & $\mathrm{N}$ & $\mathrm{N}$ & $\mathrm{A}$ \\
ApeO-KS & $\mathrm{S}$ & $\mathrm{N}$ & $\mathrm{S}$ & $\mathrm{F}$ & $\mathrm{A}$ & $\mathrm{F}$ & $\mathrm{G}$ & $\mathrm{G}$ & $\mathrm{N}$ & $\mathrm{N}$ & $\mathrm{T}$ \\
act-KS & $\mathrm{T}$ & $\mathrm{V}$ & $\mathrm{G}$ & $\mathrm{S}$ & $\mathrm{G}$ & $\mathrm{F}$ & $\mathrm{G}$ & $\mathrm{G}$ & $\mathrm{F}$ & $\mathrm{Q}$ & $\mathrm{S}$ \\
human FAS-KS & $\mathrm{I}$ & $\mathrm{N}$ & $\mathrm{S}$ & $\mathrm{F}$ & $\mathrm{G}$ & $\mathrm{F}$ & $\mathrm{G}$ & $\mathrm{G}$ & $\mathrm{S}$ & $\mathrm{N}$ & $\mathrm{V}$ \\
DEBS1 M1 KS & $\mathrm{V}$ & $\mathrm{S}$ & $\mathrm{S}$ & $\mathrm{F}$ & $\mathrm{G}$ & $\mathrm{I}$ & $\mathrm{S}$ & $\mathrm{G}$ & $\mathrm{T}$ & $\mathrm{N}$ & $\mathrm{A}$ \\
DEBS1 M2 KS & $\mathrm{V}$ & $\mathrm{S}$ & $\mathrm{A}$ & $\mathrm{F}$ & $\mathrm{G}$ & $\mathrm{V}$ & $\mathrm{S}$ & $\mathrm{G}$ & $\mathrm{T}$ & $\mathrm{N}$ & $\mathrm{A}$ \\
DEBS3 M5 KS & $\mathrm{V}$ & $\mathrm{S}$ & $\mathrm{S}$ & $\mathrm{F}$ & $\mathrm{G}$ & $\mathrm{I}$ & $\mathrm{S}$ & $\mathrm{G}$ & $\mathrm{T}$ & $\mathrm{N}$ & $\mathrm{A}$ \\
DEBS3 M6 KS & $\mathrm{V}$ & $\mathrm{S}$ & $\mathrm{A}$ & $\mathrm{F}$ & $\mathrm{G}$ & $\mathrm{V}$ & $\mathrm{S}$ & $\mathrm{G}$ & $\mathrm{T}$ & $\mathrm{N}$ & $\mathrm{A}$
\end{tabular}

b

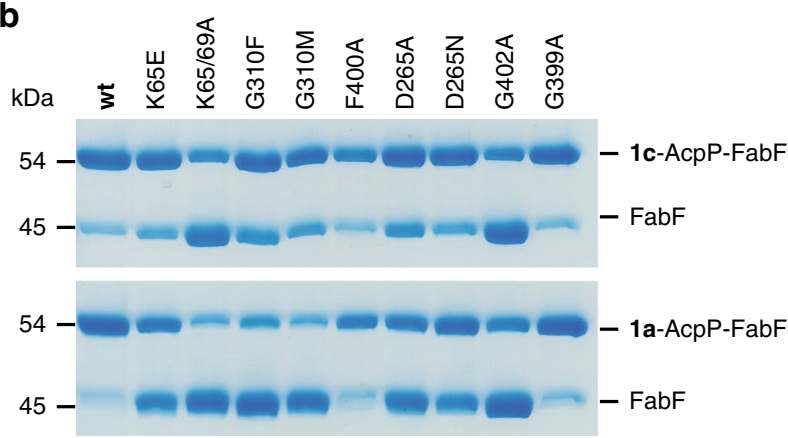

d

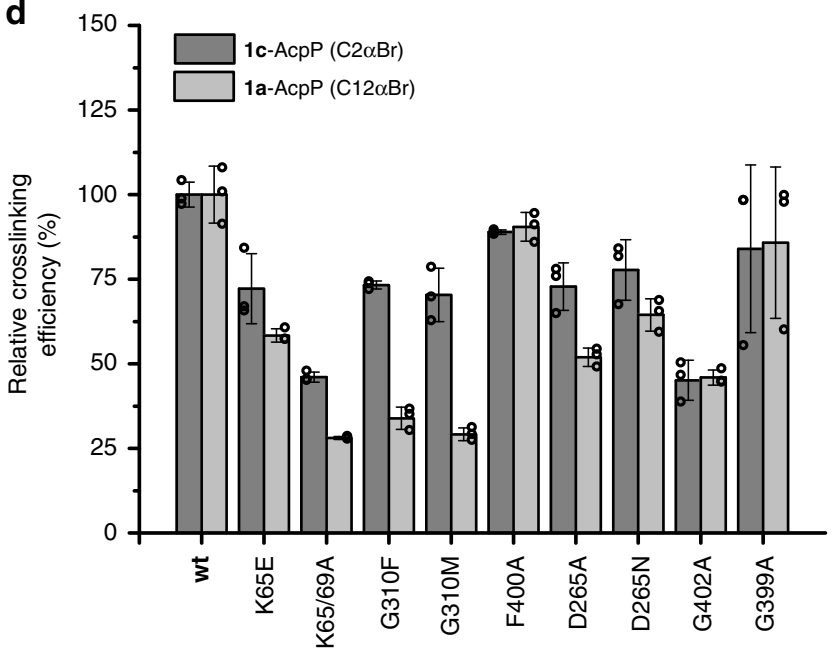

\begin{tabular}{rlllllllllllll} 
& \multicolumn{111}{c}{ Loop 2} \\
E. coli FabF & S & D & A & Y & H & M & T & S & P & P & E & N \\
Iga11-KS & C & D & A & A & H & P & T & A & P & N & R & D \\
ApeO-KS & S & D & A & W & H & M & S & A & P & H & P & E \\
act-KS & C & N & A & Y & H & M & T & G & L & K & A & D \\
human FAS-KS & T & D & G & F & K & Q & G & V & T & F & P & S \\
DEBS1 M1 KS & S & D & G & A & S & N & G & L & S & A & P & N \\
DEBS1 M2 KS & Q & D & G & A & S & N & G & L & S & A & P & S \\
DEBS3 M5 KS & Q & D & G & A & S & N & G & L & T & A & P & S \\
DEBS3 M6 KS & Q & D & G & A & T & N & G & L & A & A & P & S
\end{tabular}

C

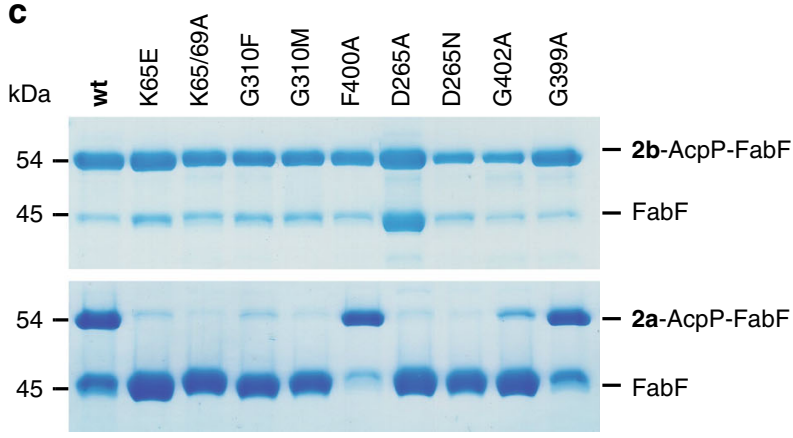

e

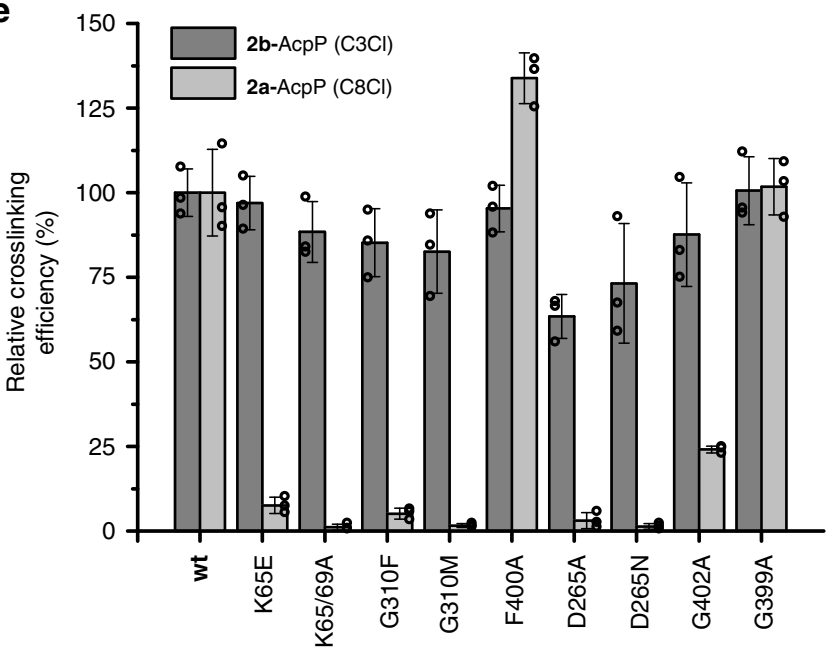

Fig. 6 Loop 1 and 2 Sequence conservation and mutagenesis. a Sequence alignment of loops 1 and 2 of FabF with representative KS sequences from noniterative type II PKS (Iga11, ApeO), iterative type II PKS (act-KS), type I FAS (Human-FAS-KS), and type I PKS (DEBS KSs). The putative gating residue (Phe400 in FabF) for each synthase is outlined in a black box. b Single time point crosslinking gels of 1c-AcpP (C2 $\alpha B r-A c p P)$ and $\mathbf{1 a}-A c p P(C 12 \alpha B r-A c p P)$ with FabF gating mutants. c Single time point crosslinking gels of $\mathbf{2} \mathbf{b}-\mathrm{AcpP}(\mathrm{C} 3 \mathrm{Cl}-\mathrm{AcpP})$ and $\mathbf{2 a}-\mathrm{AcpP}(\mathrm{C} 8 \mathrm{Cl}-\mathrm{AcpP})$ with FabF gating mutants. d Densitometric analysis of single time-point crosslinking efficiency of 1c-AcpP (C2 $\alpha B r-A c p P)$ and $\mathbf{1 a}-A c p P(C 12 \alpha B r-A c p P)$ with FabF mutants. e Densitometric analysis of single time-point crosslinking efficiency of $\mathbf{2} \mathbf{b}-\mathrm{AcpP}$ (C3Cl-AcpP) and $\mathbf{2 a}-\mathrm{AcpP}(\mathrm{C} 8 \mathrm{Cl}-\mathrm{AcpP})$ with FabF mutants. All crosslinking experiments from $\mathbf{b}$ and $\mathbf{c}$ were performed as biologically independent experiments $(n=3)$ and all data represented in $\mathbf{d}$ and $\mathbf{e}$ are the average crosslinking efficiency of each mutant normalized to the average of wt FabF crosslinking efficiency. The error bars in $\mathbf{d}$ and $\mathbf{e}$ are represented as standard deviation $( \pm S D)$ and the individual normalized measurements from each independent experiment are overlaid on top of the associated bar plot as open circles. Source data for all experiments are provided as a Source Data file.

that utilizes $a$-bromo and chloroacrylate mechanistic crosslinkers with different fatty acid chain lengths as substrate mimetics ${ }^{39}$ (Fig. 2a). We hypothesized that our panel of FabF gating mutants would generally retain wild-type (wt) activity toward smaller $\mathrm{C} 2$ and C3 chain length crosslinkers, $\mathbf{1 c}$ and 2a, but would crosslink less efficiently with longer $\mathrm{C} 8$ and $\mathrm{C} 12$ chain length crosslinkers, $\mathbf{2 b}$ and 1a, which would require transitions between gate conformations to access the KS active site.

Before assaying these gating mutants, we first evaluated the crosslinking activity of a gate removal mutant, F400A. The results show that both F400A and wt FabF crosslink with generally equal efficiency with short- and long-chain acyl-AcpP substrates (Fig. 6). Interestingly, the F400A mutant underwent crosslinking with the $\mathrm{C} 8 \mathrm{Cl}$ (2b) with higher relative efficiency to wt (133\%) both in our single time point assay and at a faster rate in our time-course crosslinking studies (Supplementary Figs. 6-11). These results suggest that mutating Phe400, a key gating residue $^{27}$, to alanine enhances the accessibility of probe to the $\mathrm{KS}$ active site cysteine ${ }^{27}$. In addition, we prepared two FabF interface mutants, $\mathrm{K} 65 \mathrm{E}$ and $\mathrm{K} 65 \mathrm{~A} / \mathrm{K} 69 \mathrm{~A}$, to demonstrate that crosslinking can also report on PPIs. Both of these mutants showed reduced crosslinking towards all probes, but crosslinking 
efficiency was most significantly reduced with long-chain crosslinkers (Fig. 6). These results suggest that well-coordinated PPIs are important for chain-flipping ${ }^{53}$ substrates into the KS active site.

To prevent loop 1 transitions from the closed to open conformation, we mutated FabF's Gly310 to a phenylalanine or methionine residue to introduce a bulky amino acid at a position that would occlude the pocket occupied by Phe 400 in the open conformation (pocket-blockage, Supplementary Fig. 12). Interestingly, G310F and G310M crosslink with 73 and 70\% efficiency with $\mathrm{C} 2 \alpha \mathrm{Br}$ (1c) but only 33 and $30 \%$ efficiency with $\mathrm{C} 12 \alpha \mathrm{Br}$ (1a), respectively. Differential crosslinking activity between short and long substrates was more pronounced for the chloroacrylate crosslinkers as G310F and G310M crosslinked with 85 and $82 \%$ efficiency with $\mathrm{C} 3 \mathrm{Cl}$ (2a) but only 5 and $1 \%$ efficiency with $\mathrm{C} 8 \mathrm{Cl}$ (2b), respectively (Fig. 6). These results indicate that blocking the open conformation reduces FabF's ability to accept longer acylACP substrates but not that of smaller substrates that more closely resemble malonyl-AcpP.

Similar patterns emerge when examining the crosslinking efficiencies of our D265A and D265N mutants of loop 2. These mutants are hypothesized to disfavor the open conformation by replacing the ion-dipole interactions involving the conserved Asp265 from loop 2 with weaker interactions. These mutations should therefore disrupt the conserved D265 coordinated hydrogen-bonding network between loops 1 and 2 that stabilizes the open conformation. D265A and D265N mutants crosslinked with 72 and $77 \%$ efficiency with $\mathrm{C} 2 \alpha \mathrm{Br}$ (1c) and 51 and $64 \%$ efficiency with $\mathrm{C} 12 \alpha \mathrm{Br}$ (1a), respectively. Furthermore, D265A and $\mathrm{D} 265 \mathrm{~N}$ crosslinked with 63 and $73 \%$ efficiency with $\mathrm{C} 3 \mathrm{Cl}$ (2a) but only 2 and $1 \%$ efficiency with $\mathrm{C} 8 \mathrm{Cl}(\mathbf{2 b})$, respectively.

In contrast, loop flexibility-modulated mutants, G399A and G402A, did not demonstrate pronounced differences in crosslinking relative to wt FabF in our assay. The G399A mutant reacted with all crosslinkers with similar efficiency as wt FabF, while crosslinking efficiencies of the G402A mutant only showed differential crosslinking activity when comparing $\mathrm{C} 3 \mathrm{Cl}$ (2a) (87\%) and C8Cl (2b) (24\%) (Fig. 6). G399A also crosslinked similarly to wt FabF in our time-course crosslinking assay (Fig. S5). While it was expected that the G399A mutant would show a difference in crosslinking efficiency, it is important to note that such a substitution is observed in nature, as the KSs from type II polyene synthases, Iga $11^{50,51}$ and $\mathrm{ApeO}^{52}$, both have alanine at this position (Fig. 5a). In addition, the dihedral angles of G399A in both the closed and open conformation are not in the Ramachandran forbidden zone (Fig. 5b, c) and can therefore be reasonably accessed by the G399A mutant.

MD simulations support a KS gating mechanism. Using these AcpP-KS crystal structures as initial coordinates, we performed $\mathrm{MD}$ simulations of $\mathrm{C} 10, \mathrm{C} 12$, and $\mathrm{C} 16-\mathrm{AcpP} \cdot \mathrm{KS}$ complexes, where "." denotes a noncovalent complex (Supplementary Figs. 1 and 13), to probe the dynamic properties of the gating processes in acyl-AcpP.KS complexes. Models of the ACP-bound acylAcpP.KSs were constructed by manually replacing the crosslinkers present in the crystal structures with the natural saturated substrates encountered by the KSs (Online Methods). In addition, simulations of $a p o-\mathrm{FabB}$ and $a p o-\mathrm{FabF}$ were performed using previously reported crystal structures (PDB: 2VB954 and $2 \mathrm{GFW}^{43}$, respectively) as initial coordinates. Lastly, in an attempt to computationally visualize the transition between the open and closed conformations of loops 1 and 2, we performed additional $\mathrm{MD}$ simulations of $a p o-\mathrm{FabF}$ and $a p o-\mathrm{FabB}$ using KS structures prepared by removing the AcpP monomers from the AcpP-KS complexes reported herein. We distinguish these structures from the crystallographic apo-FabB and apo-FabF structures by referring to them as apo-FabB* and apo-FabF*. Because these apostructures feature their loops in conformations that may not be stable in the absence of an associated acyl-ACP, we hypothesized that they may more readily undergo conformational transitions. Each of the ten systems described above was subjected to three independent $500 \mathrm{~ns}$ MD simulations. Replicates were initiated using different velocities and a total of $15 \mu$ s of MD data were collected. General analysis for all simulated systems is provided in Supplementary Figs. 14-27 and additional discussion regarding substrate interactions is provided in Supplementary Notes 6 and 7.

From this data, no transition between the closed and open loop conformations was sampled during the course of any simulation, suggesting that the coordinated movement of loops 1 and 2 between their open and closed states is a relatively slow process, likely occurring on timescales of tens or hundreds of $\mu s^{55}$. Nonetheless, analysis of the per residue backbone and side chain root mean square fluctuations (RMSFs) of the loop residues, shown in Fig. $7 \mathrm{~d}-\mathrm{i}$ and Supplementary Figs. 28-30, suggests the following: Firstly, while the loops of $a p o-F a b B$ and $a p o-F a b F$ have similar RMSFs, the loops of apo-FabF* and the acyl-AcpP.FabF complexes show greater fluctuations than do those of apo-FabB* and the acyl-AcpP.FabB complexes, respectively. Of the structures shown in Fig. $7 \mathrm{~d}-\mathrm{i}, a p o-\mathrm{FabF}^{*}$ is the most significant. RMSF analysis shows that loop 2 of apo-FabF* fluctuates more in this structure than any other simulated structures. Despite this dynamism, apo-FabF*'s loop 1 remains fairly rigid. It is possible that the transition of loop 2 from its open to closed conformation first requires the motion of loop 1 toward the active site. In fact, this movement of loop 1 would restore the oxyanion hole absent in the C16AcpP-FabF structure (Fig. 3). Therefore, the large RMSFs observed for apo-FabF*s loop 2 reveal a so-called frustrated loop unable to assume its preferred open conformation in the absence of acyl-AcpP (Fig. 7). These findings, along with published studies of PKS ACPs ${ }^{16}$, suggest that ACPs may not only be responsible for substrate delivery but also may induce loop motions that promote catalysis through allostery.

Identification of this frustrated loop suggests that the motions of loops 1 and 2 are correlated. To evaluate this hypothesis, we determined the average correlations of the motions of residues of loops 1 and 2 sampled during the course of the MD simulations of apo-KS, apo-KS*, and acyl-AcpP-KS structures. Both FabB and FabF appear to form the same general network of interloop interactions to choreograph loop motion (Fig. 8). Nonetheless, the co-relatedness of the loops are distinct in the open (Fig. 8c, e) and closed (Fig. 8a-d) states, with a greater correlation found in the closed conformation. Furthermore, the average correlation of loop 1 and 2 residues is slightly greater in simulations of acyl-AcpP-FabF complexes than simulations of either apo-FabF or $a p o-\mathrm{FabF}^{*}$. On the basis of this analysis, it is evident that loops 1 and 2 move in concert with one another and that the GFGG hinge motif is found to be most correlated to the highly conversed Asp265 residue of loop 2. Interestingly, these motifs are more highly correlated in FabF than in FabB, although higher positive average correlations between Asp265 and intra-loop 2 residues Pro271, Ser272, and Gly273 are observed in simulations of apoFabB and its acyl-AcpP complexes.

The residues of loop 1 and 2 of FabB and FabF, regardless of enzyme state, that are most strongly correlated are residues of the GFGG motif of loop 1 and Asp265 of loop 2. In fact, the closed and open conformations of these loops can be clearly distinguished by differences in these intra- and interloop interactions. That we do not observe changes in these networks of interactions within any KS simulation indicates the stability of the open and closed states. According to simulation data, the 


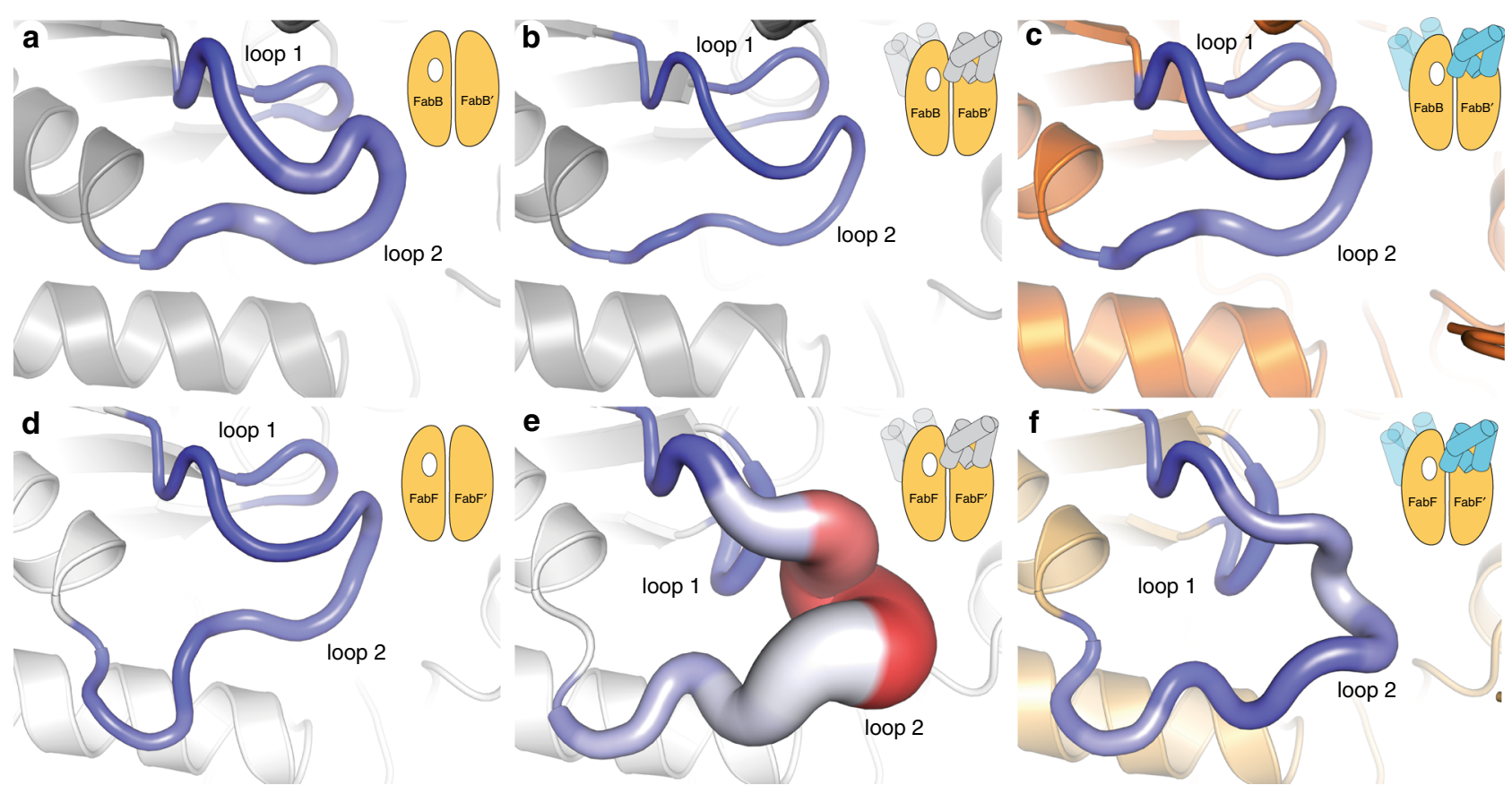

Fig. 7 Loop dynamism sampled via computer simulations. Per residue root mean square fluctuations (RMSFs) of a, apo-FabB ${ }_{2} ; \mathbf{b}$ apo-FabB ${ }^{\star}$ ( $a p o$ structure derived from $\mathrm{AcpP}_{2}-\mathrm{FabB}_{2}$ structure); c $\mathrm{AcpP}_{2} \cdot \mathrm{FabB}_{2} ; \mathbf{d}$ apo-FabF $;$; e apo-FabF ${ }_{2}$ (apo structure derived from AcpP $\mathrm{F}_{2}-\mathrm{FabF}_{2}$ structure);

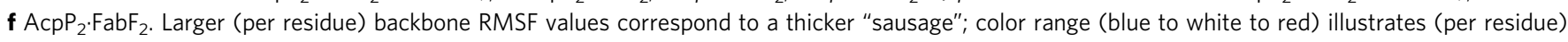
side chain RMSFs with the blue-to-red color range indicating small-to-large RMSF values.

open conformation (apo-FabF* and acyl-AcpP-FabF) is characterized by hydrogen-bonding interactions involving the GFGG motif and Asp265 and Asn404. Namely, Gly399 forms a hydrogen bond with Asn404 while the negatively charged Asp265 residue simultaneously coordinates Asn404 and the backbone amides of all residues of the GFGG motif. In the open conformation of FabF, average distances of 5.09 $\AA$, $3.91 \AA$, $3.66 \AA$, and $4.12 \AA$, respectively, are computationally observed between the center of mass of the carboxylate moiety of Asp265 and the backbone amide nitrogens of Gly399, Phe400, Gly401, and Gly402 (Fig. 9). Given the highly conserved nature of Asp265 and its substitution with a less potent hydrogen-bond acceptor (e.g., Asn265 in actKS) in iterative type II KSs, it is reasonable to postulate that the coordination of the motion of loop 1 and loop 2 is mediated by Asp265.

\section{Discussion}

KSs catalyze multi-step reactions that require the association and dissociation of two distinct acyl-AcpPs and involve the intermediacy of an acyl-enzyme adduct. Enzymes that catalyze such complex chemical transformations often implement gating mechanisms to control solvent access, exert mechanistic selectivity, and/or control reaction order ${ }^{37}$. Zhang et al. previously showed that Phe400 of Streptococcus pneumoniae FabF (SpFabF) acts as a KS gating residue ${ }^{27}$. The F400A mutant of SpFabF has reduced catalytic activity relative to wt for its native reaction, but efficiently produces triacetic acid lactone, a common shunt product of condensing enzymes that results from the mispriming and extension of malonyl-ACP. These results suggest a compromised gating system that no longer controls reaction order. The proposed gating role of Phe 400 is also supported with previously published structural data $27,43,56$. In structures of $a p o-F a b B$ and apo-FabF, Phe400 initially blocks access to the active site cysteine but after transacylation rotates away to form the malonyl-ACP binding site for the pong half-reaction $27,43,56$.
Results reported herein not only confirm and build upon the role of Phe 400 as a KS gating residue but also provide additional context for its mechanism and regulation. With this information, we propose that elongating $\mathrm{KSs}$ use a double drawbridge-like gating mechanism ${ }^{37}$ to direct substrate binding and control the timing and sequence of the transacylation and condensation halfreactions. From this study we propose the model shown in Fig. 10. Here, ACP binds to apo-KS, upon which ACP chainflips $^{53}$ its cargo into the KS active site as KS loops 1 and 2 move in a coordinated manner to assume an open conformation, forming a transient, hydrophobic delivery/extraction pocket that temporarily accommodates the substrate (Fig. 10b). Loops 1 and 2 then return to their closed conformations, directing the substrate's acyl chain into the acyl binding pocket while simultaneously restoring the oxyanion hole that facilitates transacylation (Fig. 10c). Upon transacylation, holo-ACP dissociates and Phe400 rotates to form the malonyl-ACP binding pocket ${ }^{22}$ (Fig. 10d).

The proposed gating mechanism coordinated by loops 1 and 2 is in agreement with our mutagenesis studies, MD simulations, and MSA analysis. FabF pocket-blockage mutants, G310M and G310F, and loop 2 destabilization mutants, D265A and D265N, show reduced crosslinking with long-chain acyl-AcpP substrates as compared with short-chain substrates. These results, taken together, indicate that blocking transitions to the gate-open conformation or disfavoring the gate-open conformation by disruption of the the interloop interaction network inovlving impairs KS activity with acyl-AcpPs. Interrogation of these trapped catalytic states using long time-scale MD simulations demonstrates that the open and closed conformations of FabF are stable and coordinated by a conserved network of hydrogenbonding interactions. Furthermore, this network of interactions serves to correlate the motions of loops 1 and 2 and is generally conserved in elongating KSs in type I PKS, type I FAS, and type II PKS.

Given our gating model (Fig. 10), we now propose a general mechanism used by these synthases to regulate substrate processing 
a

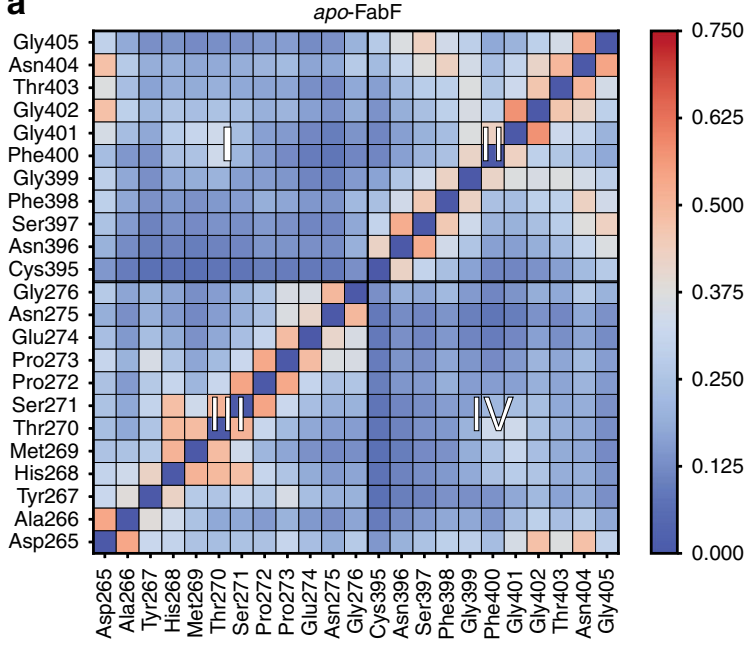

C

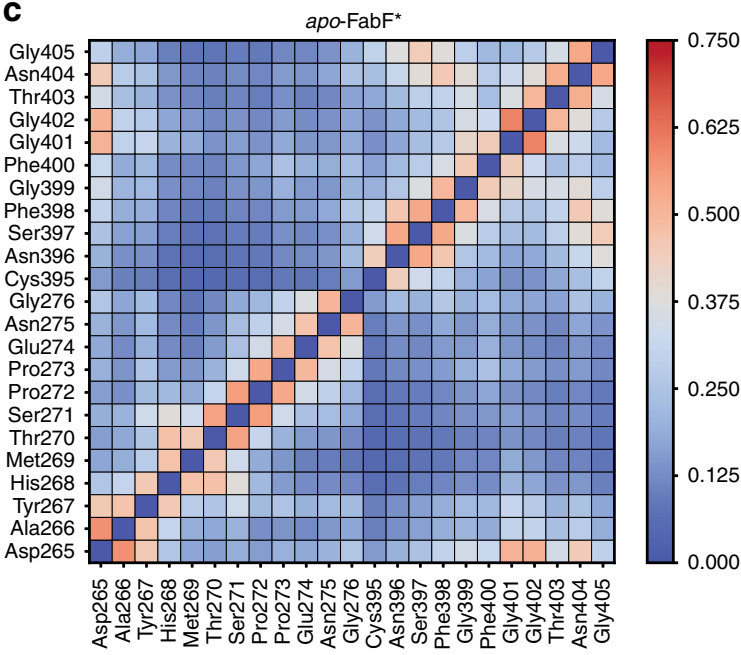

e

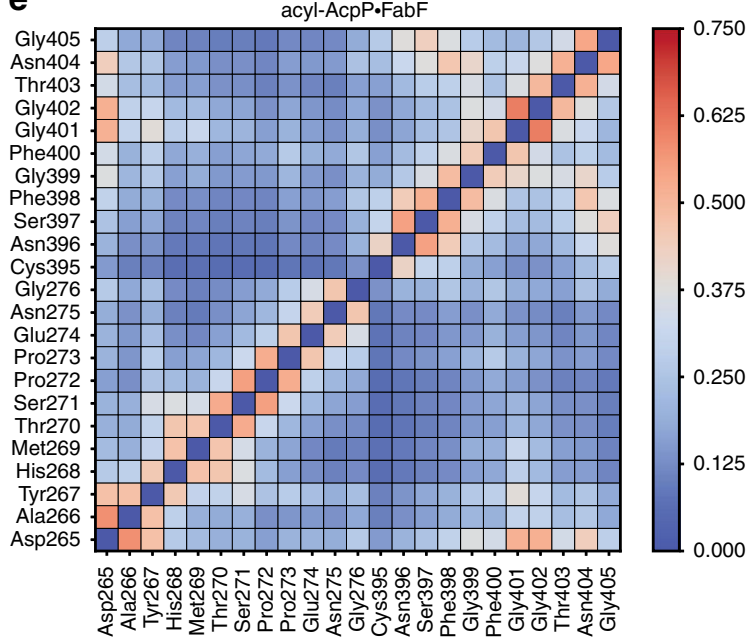

b

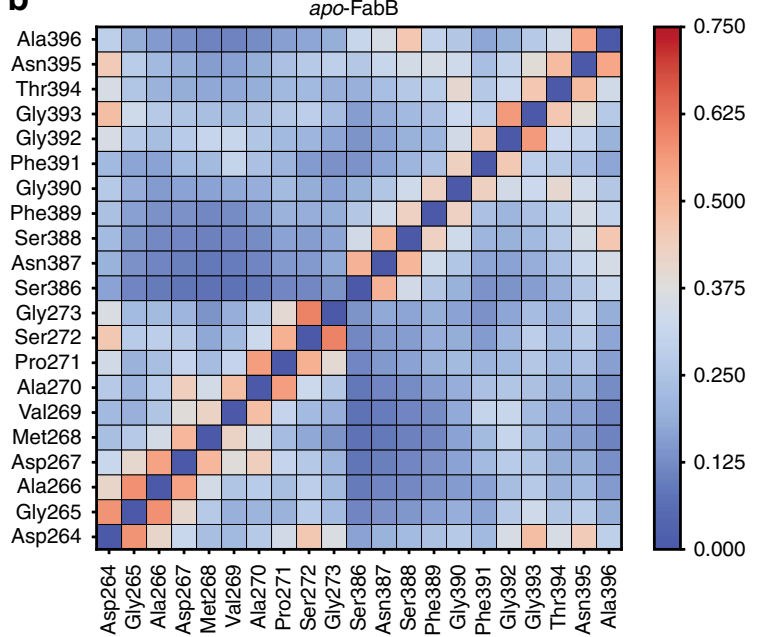

d

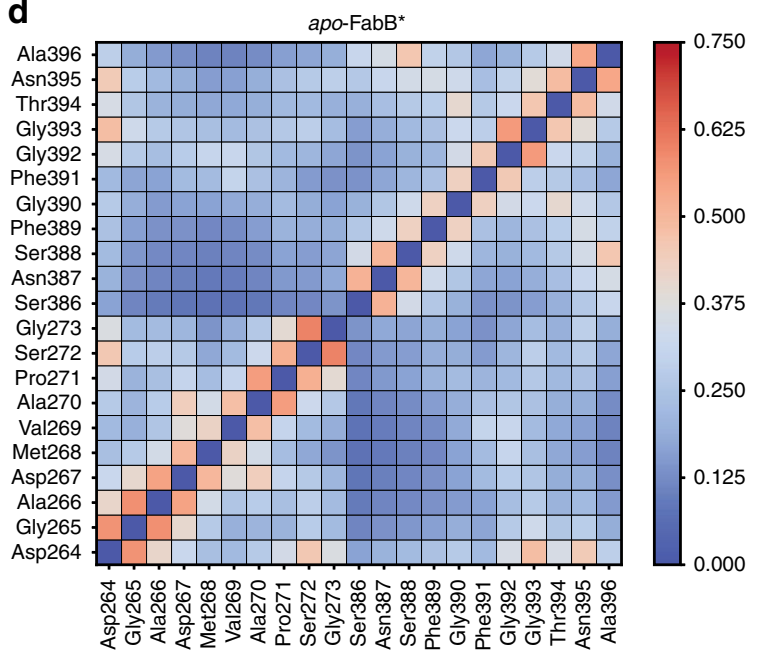

f

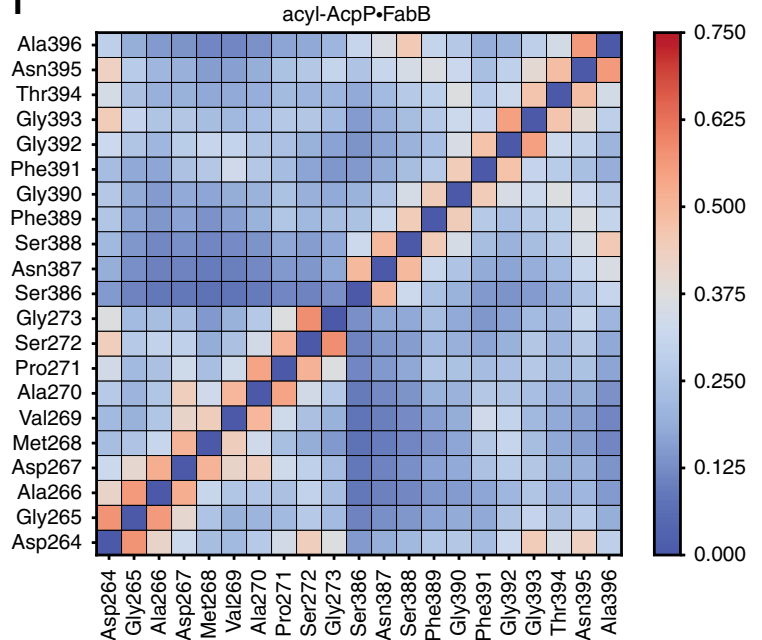

Fig. 8 Average cross correlations of the motions of key loop 1 and $\mathbf{2}$ residues sampled computationally. Cross correlations were determined by computing a motion vector for each residue of loop 1 and loop 2 from its prior position to its current over the course of each simulation. In this manner, motion vectors for each pair of residues of loop 1 and 2 are calculated for each frame of simulation data. Heat maps generated from the cross-correlation analysis of the motions of loop 1 and 2 residues of $\mathbf{a}$ apo-FabF, b apo-FabB, $\mathbf{c}$ apo-FabF*, $\mathbf{d}$ apo-FabB ${ }^{\star}$, e acyl-AcpP.FabF, and $\mathbf{f}$ acyl-AcpP.FabB, respectively, sampled using molecular dynamics (MD) simulations. a The heat map has been divided into four quadrants; quadrants II and III illustrates cross correlations of the motions of residues within loops 1 and 2, respectively. Inter-loop cross correlations are shown in quadrants I and IV. Note that each heatmap is symmetric about the diagonal that bisects quadrants II and III. 
a

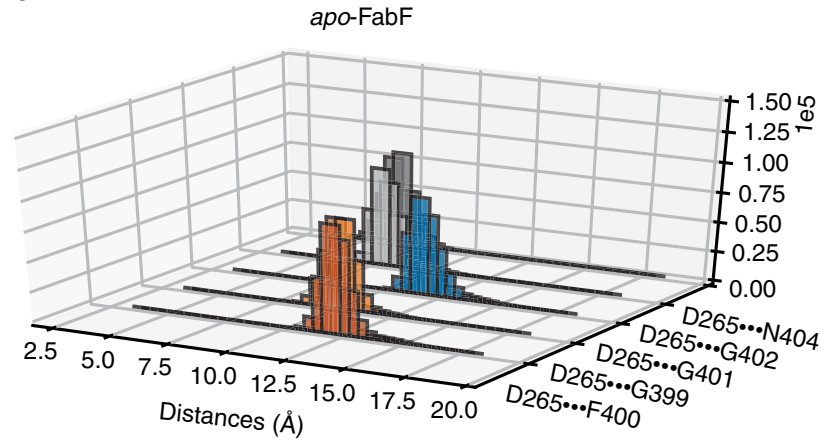

C

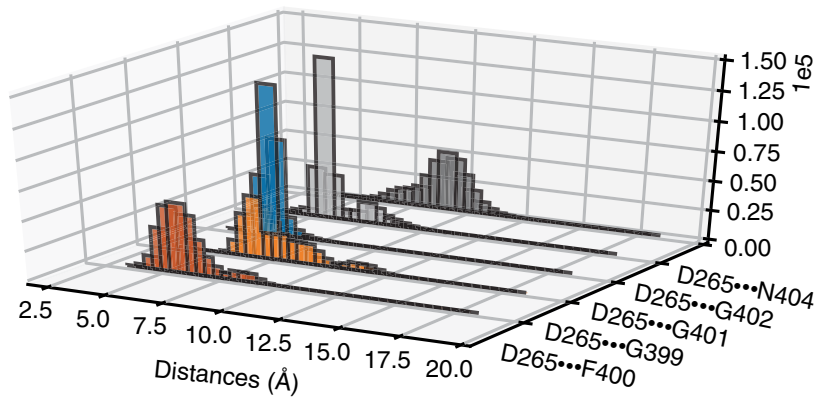

e

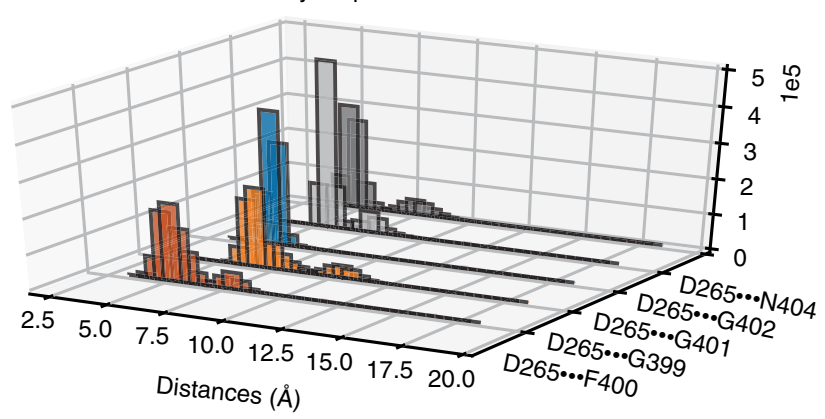

b

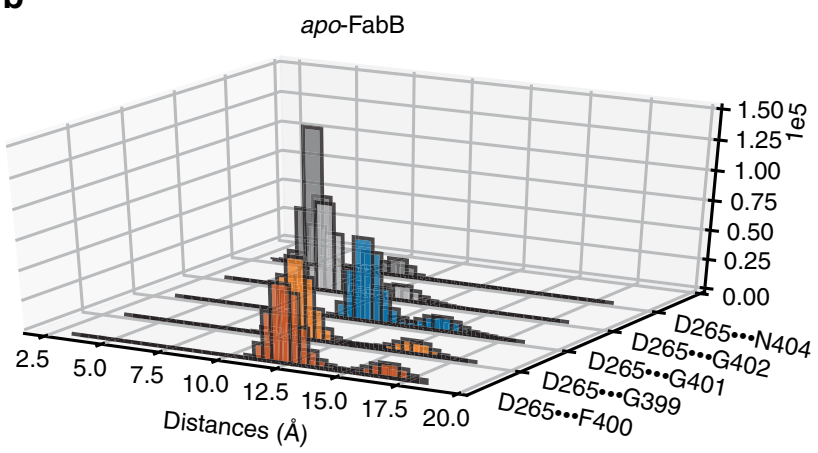

d

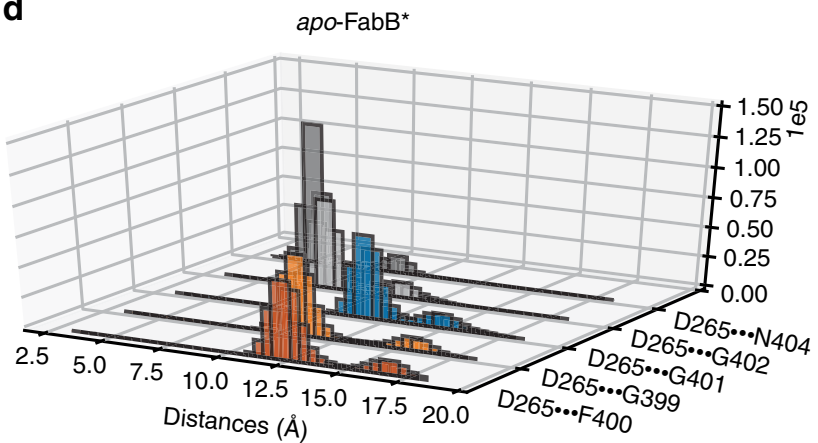

$\mathbf{f}$

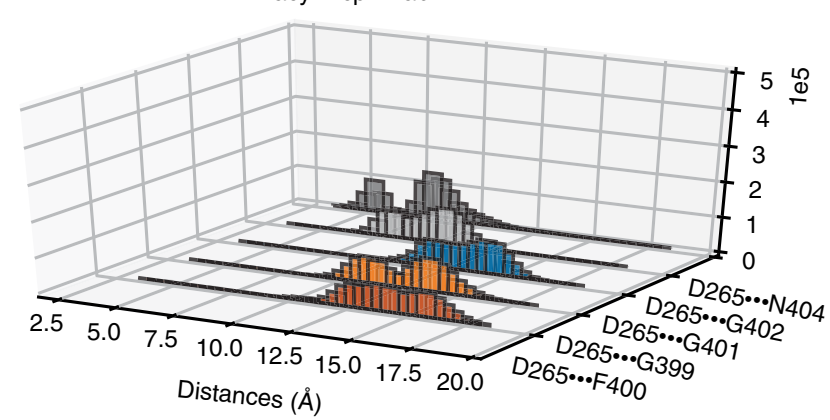

Fig. 9 Analysis of the fluctuations of inter-loop hydrogen bonding networks. Histograms showing the distances between the hydrogen-bond donating Asp265 and highly conversed (hydrogen-bonding accepting) resudes, Gly399, Phe400, Gly401, Gly402, and Asn404 sampled computationally. Histograms of these distances sampled in molecular dynamics (MD) simulations of a apo-FabF, b apo-FabB, c apo-FabF*, d apo-FabB ${ }^{\star}$, e acyl-AcpP.FabF, and $\mathbf{f}$ acyl-AcpP.FabB, respectively.

and catalysis. Transition to the open-conformation disrupts the oxyanion hole, which suggests that (trans)acylation of the active site cysteine occurs after the gate adopts the closed conformation. Consequently, the gate could act as a sensor that only reorganizes the oxyanion hole when the correct substrate is bound. Therefore, the gate and F400 may prohibit FAS KSs from reacting with fatty acyl intermediates that are not appropriate condensation substrates (i.e., $\beta$-hydroxy-acyl-ACPs).

A careful consideration of loops 1 and 2 and their potential specific functions reveals some interesting insights as well. Ramachandran analyses of the GFGG motif's fluctuations over the course of MD simulations illustrate the rigidity of loop 1 in both the closed and open conformations. The $\varphi$ and $\psi$ angles of the GFGG residues sampled computationally are narrowly distributed with hinge residues, Gly399 and Gly402, possessing distinct backbone dihedral angles in the open and closed conformations (Fig. 5b, c). This is in contrast to Ramachandran analysis of the hinge residues in loop 2, which shows that although the $\varphi$ and $\psi$ angles of Pro272 and Pro273 are distinct in the open and closed states in our crosslinked structures, they sample similar $\varphi, \psi$ angle distributions during simulations (Fig. 5e, f and Supplementary Figs. 31 and 32). These results, along with the increased sequence variation in different condensing enzyme families, suggests loop 2 may modulate loop 1 gating events, potentially conferring different activities or substrate specificities to distinct KSs. This is an appealing hypothesis, as loop 2 sits on top of loop 1, effectively capping it in when in the closed conformation. Furthermore, the dynamism of loop 2 in our apo-FabF* simulations indicates that acyl-AcpP substrates stabilize the gate-open conformation. In fact, AcpP binding or the chain flipping of acyl cargo into the KS active site may serve to trigger gating events by stabilizing loop 2's open position. Movement of loop 2 would thereby allow loop 1 to assume the gate-open conformation, raising the drawbridge and allowing substrate access to the active site. While more work is needed to test this hypothesis, a recent study by Robbins et al. demonstrated that mutation of S315A from loop 2 of DEBS KS1 increased the formation of propionate, which results from the decarboxylation of the methylmalonyl-ACP substrate ${ }^{57}$. These findings suggest an important role for loop 2 residues in DEBS and that the altered 

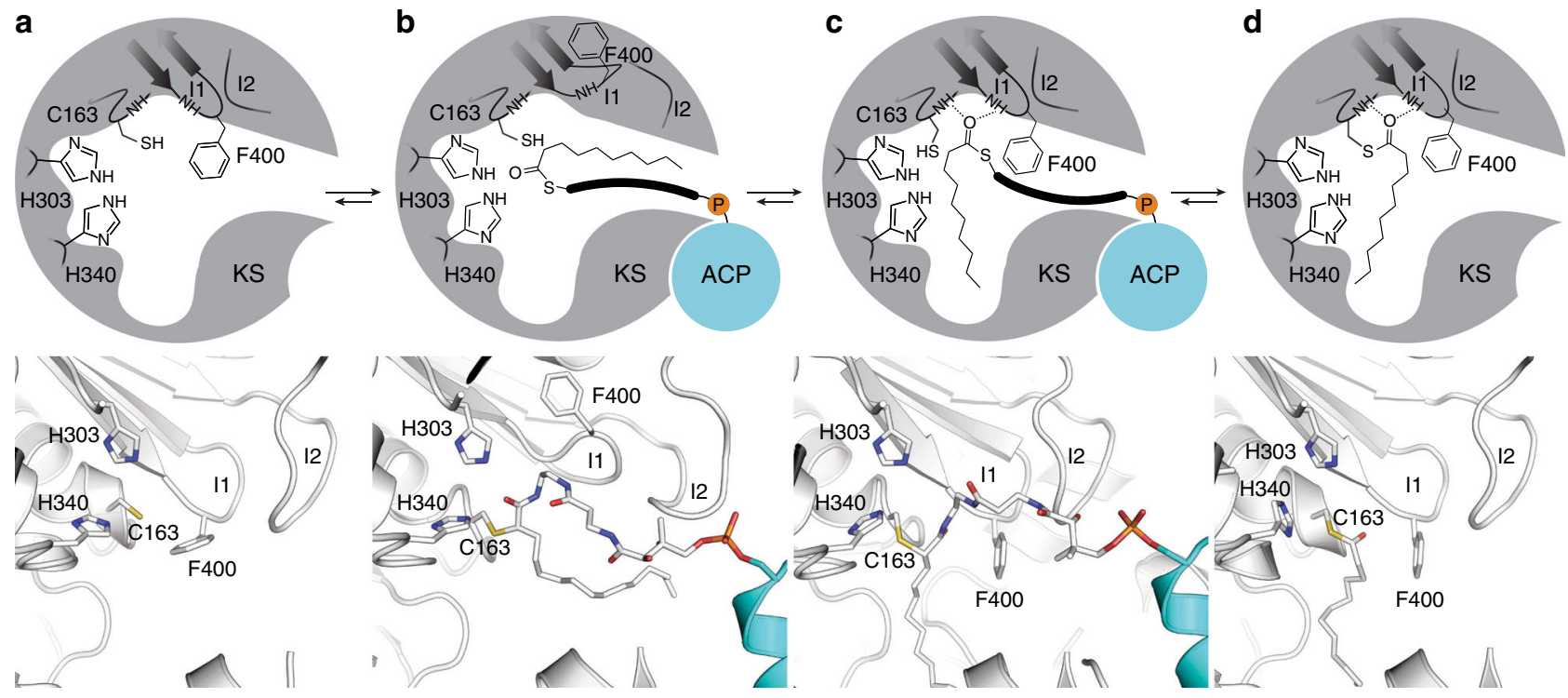

Fig. 10 Proposed gating mechanism of elongating ketosynthases. Figure demonstrates the active site conformational changes that facilitate acyl-chain transfer in elongating KSs as demonstrated by trapped crystallographic states from this work and previous studies. a The apo-form of the KS active site illustrated by a 2D schematic of the active site architecture (top) and the crystal structure of apo-FabF (PDB: 2GFW) (bottom). b Active site reorganization to the gate-open conformation upon association of acyl-AcpP with $\mathrm{KSs}$ as seen in the C16AcpP-FabF structure reported herein with a 2D schematic of the active site (top) and 3D rendering of the active site (bottom). c Active site of C12AcpP-FabB, illustrating the gate-closed transacylation competent form of the active sites of elongating KSs shown as 2D schematic (top) and 3D rendering (bottom). Residues are represented in FabF numbering. $\mathbf{d}$ The acylenzyme adduct form of FabF (PDB: 2GFY) shown as a 2D schematic (top) and 3D rendering (bottom). Active site catalytic residues Cys163, His303, His340, and Phe400 are provided in FabF residue numbering, represented as sticks, and colored according to element (C-white, O-red, N-blue, S-yellow). Hydrogen-bonding interactions are represented as dotted lines.

S315A mutant activity may be indicative of an impaired gating system.

The importance of KS-catalyzed carbon-carbon bond formation in biosynthetic pathways cannot be overstated. These enzymes perform complex reactions and employ poorly understood biochemical regulatory mechanisms to ensure substrate specificity and product fidelity. Here, the combination of chemical, structural, and computational biology together support a gating mechanism used by elongating KSs to recognize and process acyl-ACP substrates. These results provide broadly applicable insights into KS activity, function, and substrate selectivity as well as further our understanding of the E. coli AcpP interactome.

\section{Methods}

Ketosynthase production, purification, and tag cleavage. The $\mathrm{N}$-terminal $\mathrm{His}_{8}$ tag FabF and FabB recombinant proteins were expressed in E. coli BL21 and cultured in Terrific Broth (MilliporeSigma). Cells were grown in the presence of $50 \mathrm{mg} \cdot \mathrm{L}^{-1}$ kanamycin, induced with $0.5 \mathrm{mM}$ Isopropyl $\beta$-d-1-

thiogalactopyranoside (IPTG) at $\mathrm{OD}_{600}=0.8$, and incubated at $37^{\circ} \mathrm{C}$ for $4 \mathrm{~h}$. The cells were spun down by centrifugation at $500 \times g$ for $30 \mathrm{~min}$ and the collected pellets were resuspended in lysis buffer $(50 \mathrm{mM}$ Tris, $150 \mathrm{mM} \mathrm{NaCl}, 10 \%$ glycerol, pH 8.0) along with $0.5 \mathrm{mg} \cdot \mathrm{mL}^{-1}$ lysozyme (Worthington Biochemical Corp). The pelleted cells were lysed by sonication ( $4 \mathrm{~s}$ pulses for $5 \mathrm{~min}$ ), followed by another centrifugation at $17,400 \times g$ for $30 \mathrm{~min}$ to clear the lysate. The proteins were purified using Ni-NTA resin (Thermo) in the column and were washed sequentially with wash buffer ( $50 \mathrm{mM}$ Tris, $150 \mathrm{mM} \mathrm{NaCl}, 10 \%$ glycerol, $\mathrm{pH} 8.0$ )

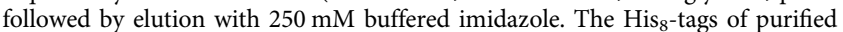
proteins were cleaved with bovine thrombin (2 $\mathrm{U}$ per $1 \mathrm{mg}$ protein) for $16 \mathrm{~h}$ at $6{ }^{\circ} \mathrm{C}$ while dialyzing against the dialysis buffer $(50 \mathrm{mM}$ Tris $\mathrm{pH} 8.0,150 \mathrm{mM} \mathrm{NaCl}, 10 \%$ glycerol, $0.5 \mathrm{mM}$ TCEP). Resulting solutions were re-purified using Ni-NTA resin (Thermo Fisher Scientific) to trap the un-cleaved proteins. The FabF and FabB were further purified by FPLC using the HiLoad Superdex 200 (GE Biosciences) size exclusion column. The eluted proteins were collected and concentrated to 2-4 $\mathrm{mg} \cdot \mathrm{mL}^{-1}$ using Amicon Ultra Centrifuge Filters (MilliporeSigma) with $10 \mathrm{kDa}$ molecular weight cut off.

Native AcpP recombinant protein purification. Native AcpP recombinant protein was expressed in E. coli BL21 (DE3) and grown in Terrific Broth (Source).
Cells were grown at $37^{\circ} \mathrm{C}$ in the presence of $100 \mathrm{mg} \cdot \mathrm{L}^{-1}$ ampicillin, induced with $0.5 \mathrm{mM}$ IPTG at $\mathrm{OD}_{600}=0.8$, and incubated at $18^{\circ} \mathrm{C}$ for $16 \mathrm{~h}$. The cells were spun down by centrifugation at $500 \times g$ for $30 \mathrm{~min}$, and the collected pellets were resuspended in lysis buffer $(50 \mathrm{mM}$ Tris, $5 \%$ glycerol, $\mathrm{pH} 7.4)$ along with 0.1 $\mathrm{mg} \cdot \mathrm{mL}^{-1}$ lysozyme (Worthington Biochemical Corp). The pelleted cells were lysed by sonication ( $4 \mathrm{~s}$ pulses for $5 \mathrm{~min}$ ). Due to the high stability of AcpP, irrelevant proteins were precipitated by dripping in same volume of isopropanol into the lysate at the speed of $0.1 \mathrm{~mL} \cdot \mathrm{s}^{-151}$. The resulting mixture was spun down by centrifugation at $11,200 \times g$ for $1 \mathrm{~h}$ and the supernatant was injected directly into FPLC. AcpP was purified by HiTrap Q HP anion exchange chromatography column in $50 \mathrm{mM}$ Tris buffer with a gradient of $\mathrm{NaCl}$ from $0 \mathrm{M}$ to $1 \mathrm{M}$ and the native AcpP eluted around $0.3 \mathrm{M} \mathrm{NaCl}$. The eluted protein was collected and concentrated using Amicon Ultra Centrifuge Filters (Millipore) with $3 \mathrm{kDa}$ molecular weight cut off.

Holo-AcpP apofication and apo-AcpP purification. The purified protein was resulted in a mixture of holo-AcpP and apo-AcpP. The phosphopantetheine moiety on holo-AcpP was removed by acyl carrier protein hydrolase $(\mathrm{AcpH})$ in lysis buffer for $16 \mathrm{~h}^{58}$. Final reaction concentrations: $5 \mathrm{mg} \cdot \mathrm{mL}^{-1}$ AcpP mixture, $0.01 \mathrm{mg} \cdot \mathrm{mL}^{-1}$ AcpH, $50 \mathrm{mM}$ Tris (pH 7.4), $10 \%$ glycerol, $10 \mathrm{mM} \mathrm{MgCl}_{2}, 5 \mathrm{mM} \mathrm{MnCl}_{2}$, and $0.25 \%$ DTT. The resulting pure apo-AcpP, was analyzed by conformationally sensitive urea PAGE (2.5 M urea), and further purified by FPLC using HiLoad 16/600 Superdex 75 PG (GE Biosciences) size exclusion column to remove AcpH. The eluted protein was collected and concentrated using Amicon Ultra Centrifuge Filters (Millipore Sigma) with $3 \mathrm{kDa}$ molecular weight cut off.

Apo-AcpP loading and crypto-AcpP purification. All crosslinkers were loaded onto apo-AcpP using a one-pot chemoenzymatic method ${ }^{38}$. This method utilizes three CoA biosynthetic enzymes (CoaA, CoaD, $\mathrm{CoaE}$ ) to form $\mathrm{CoA}$ analogues and a phosphopantetheinyl transferase (PPTase, Sfp) to load them onto apo-AcpP, resulting in crypto-AcpP. Final reaction concentrations: $1 \mathrm{mg} \cdot \mathrm{mL}^{-1}$ apo-AcpP, $0.04 \mathrm{mg} \cdot \mathrm{mL}^{-1} \mathrm{Sfp}, 0.01 \mathrm{mg} \cdot \mathrm{mL}^{-1} \mathrm{CoaA}, 0.01 \mathrm{mg} \cdot \mathrm{mL}^{-1} \mathrm{CoaD}, 0.01 \mathrm{mg} \cdot \mathrm{mL}^{-1} \mathrm{CoaE}$, $50 \mathrm{mM}$ potassium phosphate $\mathrm{pH} 7.2,12.5 \mathrm{mM} \mathrm{MgCl}_{2}, 1 \mathrm{mM}$ DTT, $0.2 \mathrm{mM}$ pantetheineamide probe (1a,1c or $\mathbf{2 a}, \mathbf{2 b}$ ) (Fig. 2), and $8 \mathrm{mM}$ ATP. The stock solutions of crosslinkers 1a-1c, $2 \mathbf{a}$, and $\mathbf{2 c}$ were prepared by dissolving them in DMSO to a final concentration of $50 \mathrm{mM}$. Reactions were incubated at $37^{\circ} \mathrm{C}$ for $16 \mathrm{~h}$ and then purified by HiLoad Superdex 75 (GE Biosciences) size exclusion column. The eluted protein was collected and concentrated using Amicon Ultra Centrifuge Filters (Millipore Sigma) with $3 \mathrm{kDa}$ molecular weight cut off up to a concentration at $1-3 \mathrm{mg} \cdot \mathrm{mL}^{-1}$. 
Crosslinking reaction and crosslinked complex purification. The crosslinking reactions were carried out by mixing crypto-AcpP with its partner protein ( $\mathrm{FabF}$ or FabB) in 5:1 ratio at $37^{\circ} \mathrm{C}$ for $16 \mathrm{~h}$. Reactions were examined by $12 \%$ SDS PAGE and purified by HiLoad 16/600 Superdex 200 PG (GE Biosciences) size exclusion column using minimal buffer ( $20 \mathrm{mM}$ Tris, $50 \mathrm{mM} \mathrm{NaCl}$, pH 7.4). The resulting protein complex was greater than $95 \%$ in purity, determined by SDS PAGE, and was concentrated to $8-10 \mathrm{mg} \cdot \mathrm{mL}^{-1}$ using Amicon Ultra Centrifuge Filters (MilliporeSigma) with $30 \mathrm{kDa}$ molecular weight cut off. The concentrated crosslinked complex was immediately used for protein crystallization or flash-frozen and stored in $-80^{\circ} \mathrm{C}$ freezer for later use.

Construction of ketosynthase mutants. Ketosynthase mutants were generated using the site directed mutagenesis method developed by Liu and Naismith ${ }^{59}$. All constructs were verified via Sanger sequencing (Eton Bioscience Inc.). Primers used for mutagenesis can be found in Supplementary Fig. 33.

Crosslinking assay. Each reaction was set up to contain $40 \mu \mathrm{M}$ of crypto-AcpP $20 \mu \mathrm{M}$ of FabF, and buffer $(25 \mathrm{mM}$ Tris, $150 \mathrm{mM} \mathrm{NaCl}, 10 \%$ glycerol, $0.5 \mathrm{mM}$ TCEP, $\mathrm{pH}$ 7.4). To stop the reaction at the designated time point, $2.5 \mu \mathrm{L}$ of the reaction solution was mixed with $10 \mu \mathrm{L}$ of $3 \mathrm{X}$ SDS dye. Samples were analyzed by $12 \%$ SDS-PAGE and the intensity of protein bands was measured by Image $]^{60}$. The crosslinking efficiency was calculated by $[($ Icrosslink $) /($ Icrosslink $+I$ FabF $)] \times 100 \%$ where " $P$ " stands for band intensity. All reactions were performed in biological triplicate and the crosslinking efficiency of mutants was normalized to the average wild type crosslinking efficiency to obtain a normalized relative crosslinking efficiency. The plotted data represent the average normalized crosslinking efficiency of each mutant and the error bars are represented as standard deviation $( \pm \mathrm{SD})$.

General synthetic methods. General synthetic methods are provided in Supplementary Note 8 and NMR characterization of compounds and intermediates is provided in Supplementary Figs. 40-43.

Crystallization, structure determination, and refinement. The crystals of the crosslinked complexes were grown by vapor diffusion at $6^{\circ} \mathrm{C}$. In detail, $1 \mu \mathrm{L}$ of crosslinked complex $\left(8-10 \mathrm{mg} \cdot \mathrm{mL}^{-1}\right)$ was mixed with $1 \mu \mathrm{L}$ of corresponding crystallographic condition and the mixture was placed inverted over $500 \mu \mathrm{L}$ of the well solution (hanging-drop method). The AcpP-FabF complexes crystallized in 26-30\% PEG $8 \mathrm{~K}, 0.1 \mathrm{M}$ sodium cacodylate $\mathrm{pH} 6.5$, and $0.3 \mathrm{M} \mathrm{NaOAc}$, yielding numerous orange rods amongst a heavy precipitate. Seeding trials were performed to increase size and quality of the samples. The C12AcpP-FabB complexes produced crystals in two conditions: $20 \%$ PEG $8 \mathrm{~K}, 0.2 \mathrm{M} \mathrm{Mg}(\mathrm{OAc})_{2}, 0.1 \mathrm{M}$ sodium cacodylate $\mathrm{pH} 6.5$ as well as $30 \%$ PEG $8 \mathrm{~K}, 0.2 \mathrm{M}\left(\mathrm{NH}_{4}\right)_{2} \mathrm{SO}_{4}, 0.1 \mathrm{M}$ sodium cacodylate, $\mathrm{pH}$ 6.5. Both conditions yielded square plates and required 1-2 weeks for complete growth of crystals. The C16AcpP-FabB complexes produced crystals in $18-24 \%$ PEG $8 \mathrm{~K}, 0.1 \mathrm{M}$ sodium cacodylate, and $0.3 \mathrm{M} \mathrm{NaOAc}$, pH 6.5 .

All data were collected at the Advanced Light Source synchrotron at Berkeley. Data were indexed using iMosflm ${ }^{61}$ then processed and scaled using aimless from the CCP4 software suite ${ }^{62,63}$. Scaled reflection output data were used for molecular replacement and model building in PHENIX ${ }^{64}$. For AcpP-FabF complexes, phases were solved using molecular replacement by first locating the larger FabF (PDB: $2 \mathrm{GFW}^{43}$ ) monomer followed by a second search function to place the smaller AcpP (PDB: 2FAC ${ }^{65}$ ) molecule in the residual density. For C12AcpP-FabB and C16AcpP-FabB complexes, initial phases were solved using molecular replacement by first performing a search function using the FabB monomer (PDB: $1 \mathrm{G}^{3} \mathrm{X}^{30}$ ) and then manually placing the AcpP molecules in the residual electron density. The parameter file for the covalently bonded $4^{\prime}$-phosphopantetheine was generated using Jligand $(\mathrm{CCP} 4)^{63}$. Manually programmed parameter restraints were used to create the associated covalent bonds between $4^{\prime}$-phosphopantetheine to Ser36 and Cys163 during refinement. These parameters are provided in the parm-file folder located in the Source Data file.

Preparation of apo-KS structures for MD simulations. Simulations of apo-FabB and $a p o$-FabF were performed using two sets of initial coordinates: one derived from previously reported $1.55 \AA$ apo-FabB (PDB: $2 \mathrm{VB} 9^{54}$ ) and $2.40 \AA$ apo-FabF (PDB: $2 \mathrm{GFW}^{43}$ ) and a second set generated by modifying the crosslinked structures of E. coli AcpP-FabB and AcpP-FabF reported herein by deleting coordinates corresponding to atoms from AcpP.

Preparation of acyl-AcpP-KS structures for MD simulations. The crosslinked structures of E. coli AcpP-FabB and AcpP-FabF reported herein were used to prepare the initial coordinates used to perform MD. The asymmetric unit of the AcpP-FabF structure is-unlike that of crosslinked AcpP-FabB-the 1:1 AcpP.FabF complex. In order to prepare coordinates for the (functional) biological unit, $\mathrm{AcpP}_{2} \cdot \mathrm{FabF}_{2}$, the asymmetric unit of the $\mathrm{AcpP}_{1}-\mathrm{FabF}_{1}$ structure was rotated about the twofold symmetry axis of the AcpP-FabF biological unit. The 2:2 complexes of acyl-AcpPs shown in Scheme S1 and either FabB or FabF were constructed by manually modifying the chemical crosslinker present the experimental structures. Modifications of this moiety were performed using
Avogadro 1.1.166 [http://avogadro.cc/], Gaussview 5.0.9, and Pymol v.1.8.6, and Pymol v.2.2.3. Schrodinger was used to add poorly resolved C- and N-terminal residues omitted in the dimeric AcpP.FabB and AcpP.FabF complexes. The protonation state of titratable residues in the apo-KSs and acyl-AcpP.KS didomains were assigned using the $\mathrm{H}++$ webserver, with the exception of the active site Cys163 of FabB and FabF; this residue was simulated in as a thiolate ${ }^{67-70}$. Histidine protonation states were inspected manually.

Parameter generation for acyl-AcpP. For each simulated AcpP.KS complex, the acyl substrate, phosphopantetheine cofactor, and the conserved serine residue of AcpP to which the cofactor is affixed were treated as a single nonstandard residue. AMBER ( $\left(\mathrm{ff}^{14 \mathrm{SB}^{71}}{ }^{7}\right.$ ) and $\mathrm{GAFF}^{72}$ type force field parameters were assigned to the atoms of these nonstandard residues using ANTECHAMBER ${ }^{73}$. Partial charges for all atoms in all nonstandard residues were determined using the RESP methodology $^{74}$. All quantum calculations were performed using Gaussian 09 (https:// gaussian.com/glossary/g09/).

Preparation of simulation cells. Proteins were solvated in a water box with TIP3 $\mathrm{P}^{75}$ water molecules. Using TLEAP ${ }^{76}$, the simulation cell was constructed such that its edges were placed $10 \AA$ away from the closest proteinogenic atom. TLEAP was used to neutralize and salt the water box to mimic physiological conditions $(0.15 \mathrm{M})$. Counterions $\left(\mathrm{Na}^{+}\right.$and $\left.\mathrm{Cl}^{-}\right)$were also added to the simulation cell randomly.

Simulation details. Amber 16 and Amber $18^{76}$ were used to perform all MD simulations. All simulations were performed using the ff $14 \mathrm{SB}^{71}$ and $\mathrm{GAFF}^{72}$ force fields. A 2 fs time-step was utilized via the SHAKE algorithm, which constrains all nonpolar bonds involving hydrogen atoms ${ }^{77}$. Long-range electrostatic interactions were treated using the Particle Mesh Ewald (PME) method with a $10 \AA$ cutoff for all non-bonded interactions ${ }^{78}$. Both solvated protein complexes were energy minimized in a two-step fashion. In a first step, solvent molecules and counterions were allowed to relax, while all protein atoms were restrained using a harmonic potential $\left(k=500 \mathrm{kcal} \mathrm{mol}^{-1} \AA^{-2}\right)$. This geometry optimization was followed by an unrestrained energy minimization of the entire system. The thermal energy available at a physiological temperature of $310 \mathrm{~K}$ was slowly added to each system over the course of a $3.5 \mathrm{~ns}$ NVT ensemble simulation. The solvated complexes were then subjected to unbiased isobaric-isothermal (NPT) simulations for $25 \mathrm{~ns}$ in order to equilibrate the heated structures. Three independent $500 \mathrm{~ns}$ production MD (NPT ensemble) of each system were performed with different initial velocities. For both NVT and NPT simulations, the Langevin thermostat $\left(\lambda=5.0 \mathrm{ps}^{-1}\right)$ was used to maintain temperature control ${ }^{79}$. Pressure regulation in NPT simulations (target pressure of $1 \mathrm{~atm}$ ) was achieved by isotropic position scaling of the simulation cell volume using a Berendsen barostat.

Analysis and visualization of simulation data. Analysis was performed using CPPTRAJ Version V4.14.0 (AmberTools V19.00), PYTRAJ v2.02.dev0, a Python front-end for the CPPTRAJ analysis code, and MDTRAJ v1.9.380,81. Trajectories were visualized using VMD 1.9.4 ${ }^{82}$ and Pymol v1.8.6 and Pymol v2.2.3. Coordinate data were written to disk every $10 \mathrm{ps}$.

Reporting summary. Further information on research design is available in the Nature Research Reporting Summary linked to this article.

\section{Data availability}

All crystallographic models and data were submitted to the Worldwide Protein Data Bank (wwPDB, www.wwpdb.org) under the accession codes 6OKC (C12AcpP-FabB), 6OKF (C16AcpP-FabB), 6OLT (C12AcpP-FabF), 6OKG (C16AcpP-FabF). All parameter files for generating and running $\mathrm{MD}$ simulations are provided in the parmfiles folder in the online Source Data file. All original source data used for crosslinking analysis reported in Fig. 6 and Supplementary Figs. 5, 6, and 8 are provided as a Source Data file. All other data are available from the corresponding authors on reasonable request.

\section{Code availability}

All MD simulation (AMBER 16 and AMBER 18) and analysis (PYTRAJ, CPPTRAJ) code used herein is published and publicly available.

Received: 8 May 2019; Accepted: 6 March 2020;

Published online: 07 April 2020

\section{References}

1. Hertweck, C. The biosynthetic logic of polyketide diversity. Angew. Chem. Int. Ed. 48, 4688-4716 (2009). 
2. Staunton, J. \& Weissman, K. J. Polyketide biosynthesis: a millennium review. Nat. Prod. Rep. 18, 380-416 (2001).

3. Beld, J., Lee, D. J. \& Burkart, M. D. Fatty acid biosynthesis revisited: structure elucidation and metabolic engineering. Mol. Biosyst. 11, 38-59 (2015).

4. White, S. W., Zheng, J., Zhang, Y.-M. \& Rock, C. O. The structural biology of type II fatty acid biosynthesis. Annu. Rev. Biochem. 74, 791-831 (2005).

5. Herbst, D. A., Townsend, C. A. \& Maier, T. The architectures of iterative type I PKS and FAS. Nat. Prod. Rep. https://doi.org/10.1039/C8NP00039E (2018).

6. Herbst, D. A. et al. The structural organization of substrate loading in iterative polyketide synthases. Nat. Chem. Biol. 14, 474-479 (2018).

7. Herbst, D. A., Jakob, R. P., Zähringer, F. \& Maier, T. Mycocerosic acid synthase exemplifies the architecture of reducing polyketide synthases. Nature 531, 533-537 (2016).

8. Maier, T., Leibundgut, M. \& Ban, N. The crystal structure of a mammalian fatty acid synthase. Science. 321, 1315 LP-1322 (2008).

9. Maier, T., Leibundgut, M., Boehringer, D. \& Ban, N. Structure and function of eukaryotic fatty acid synthases. Q. Rev. Biophys. 43, 373-422 (2010).

10. Crosby, J. \& Crump, M. P. The structural role of the carrier protein-active controller or passive carrier. Nat. Prod. Rep. 29, 1111-1137 (2012).

11. Chen, A., Re, R. N. \& Burkart, M. D. Type II fatty acid and polyketide synthases: deciphering protein-protein and protein-substrate interactions. Nat. Prod. Rep. 35, 1029-1045 (2018).

12. Kapur, $\mathrm{S}$. et al. Reprogramming a module of the 6-deoxyerythronolide $B$ synthase for iterative chain elongation. Proc. Natl Acad. Sci. 109, 4110 LP4115 (2012).

13. Masoudi, A., Raetz, C. R. H., Zhou, P. \& Pemble, C. W. IV Chasing acyl carrier protein through a catalytic cycle of lipid A production. Nature 505, 422 (2013).

14. Nguyen, C. et al. Trapping the dynamic acyl carrier protein in fatty acid biosynthesis. Nature 505, 427-431 (2014).

15. Lowry, B., Li, X., Robbins, T., Cane, D. E. \& Khosla, C. A turnstile mechanism for the controlled growth of biosynthetic intermediates on assembly line polyketide synthases. ACS Cent. Sci. 2, 14-20 (2016).

16. Jiménez-Osés, G. et al. The role of distant mutations and allosteric regulation on LovD active site dynamics. Nat. Chem. Biol. 10, 431 (2014).

17. Blatti, J. L. et al. Manipulating fatty acid biosynthesis in microalgae for biofuel through protein-protein interactions. PLoS One 7, 1-12 (2012).

18. Ruppe, A. \& Fox, J. M. Analysis of interdependent kinetic controls of fatty acid synthases. ACS Catal. 8, 11722-11734 (2018).

19. Cronan, J. E. \& Thomas, J. Bacterial fatty acid synthesis and its relationships with polyketide synthetic pathways. Methods Enzymol. 459, 395-433 (2009).

20. Jenner, M. et al. Acyl-chain elongation drives ketosynthase substrate selectivity in trans-acyltransferase polyketide synthases. Angew. Chem. Int. Ed. 54, 1817-1821 (2015).

21. Heil, C. S., Wehrheim, S. S., Paithankar, K. S. \& Grininger, M. Fatty acid biosynthesis: chain-length regulation and control. ChemBioChem 20, 2298-2321 (2019).

22. Klaus, M. \& Grininger, M. Engineering strategies for rational polyketide synthase design. Nat. Prod. Rep. 35, 1070-1081 (2018).

23. Gajewski, J. et al. Engineering fatty acid synthases for directed polyketide production. Nat. Chem. Biol. 13, 363-365 (2017).

24. Zhu, Z. et al. Expanding the product portfolio of fungal type i fatty acid synthases. Nat. Chem. Biol. 13, 360-362 (2017).

25. Fischer, M. \& Grininger, M. Strategies in megasynthase engineering-fatty acid synthases (FAS) as model proteins. Beilstein J. Org. Chem. 13, 1204-1211 (2017).

26. Gajewski, J., Pavlovic, R., Fischer, M., Boles, E. \& Grininger, M. Engineering fungal de novo fatty acid synthesis for short chain fatty acid production. Nat. Commun. 8, 1-8 (2017).

27. Zhang, Y.-M., Hurlbert, J., White, S. W. \& Rock, C. O. Roles of the active site water, histidine 303 , and phenylalanine 396 in the catalytic mechanism of the elongation condensing enzyme of streptococcus pneumoniae. J. Biol. Chem. 281, 17390-17399 (2006).

28. von Wettstein-Knowles, P., Olsen, J. G., McGuire, K. A. \& Henriksen, A. Fatty acid synthesis. FEBS J. 273, 695-710 (2006).

29. Zhang, Y.-M., Wu, B., Zheng, J. \& Rock, C. O. Key residues responsible for acyl carrier protein and $\beta$-ketoacyl-acyl carrier protein reductase (FabG) interaction. J. Biol. Chem. 278, 52935-52943 (2003).

30. Zhang, Y.-M. et al. Identification and analysis of the acyl carrier protein (ACP) docking site on $\beta$-ketoacyl-ACP synthase III. J. Biol. Chem. 276, 8231-8238 (2001).

31. Tallorin, L. et al. Trapping of the enoyl-acyl carrier protein reductase-acyl carrier protein interaction. J. Am. Chem. Soc. 138, 3962-3965 (2016).

32. Yu, X., Liu, T., Zhu, F. \& Khosla, C. In vitro reconstitution and steady-state analysis of the fatty acid synthase from Escherichia coli. Proc. Natl Acad. Sci. 108, 18643 LP-18648 (2011).

33. Dodge, G. J. et al. Structural and dynamical rationale for fatty acid unsaturation in Escherichia coli. Proc. Natl. Acad. Sci. https://doi.org/10.1073/ pnas.1818686116 (2019).
34. Milligan, J. C. et al. Molecular basis for interactions between an acyl carrier protein and a ketosynthase. Nat. Chem. Biol. 15, 669-671 (2019).

35. Marcella, A. M., Culbertson, S. J., Shogren-Knaak, M. A. \& Barb, A. W. Structure, high affinity, and negative cooperativity of the Escherichia coli holo(acyl carrier protein):holo-(acyl carrier protein) synthase complex. J. Mol. Biol. 429, 3763-3775 (2017).

36. Rafi, S. et al. Structure of acyl carrier protein bound to FabI, the FASII enoyl reductase from escherichia coli. J. Biol. Chem. 281, 39285-39293 (2006).

37. Gora, A., Brezovsky, J. \& Damborsky, J. Gates of enzymes. Chem. Rev. 113, 5871-5923 (2013).

38. Worthington, A. S. \& Burkart, M. D. One-pot chemo-enzymatic synthesis of reporter-modified proteins. Org. Biomol. Chem. 4, 44-46 (2006).

39. Worthington, A. S., Porter, D. F. \& Burkart, M. D. Mechanism-based crosslinking as a gauge for functional interaction of modular synthases. Org. Biomol. Chem. 8, 1769-1772 (2010).

40. Finzel, K., Lee, D. J. \& Burkart, M. D. Using modern tools to probe the structure-function relationship of fatty acid synthases. ChemBioChem 16, 528-547 (2015).

41. D’Agnolo, G., Rosenfeld, I. S. \& Vagelos, P. R. Multiple forms of betaketoacyl-acyl carrier protein synthetase in Escherichia coli. J. Biol. Chem. 250, 5289-5294 (1975).

42. Garwin, J., Klages, A. \& Cronan, J. Structural, enzymatic, and genetic studies of beta-ketoacyl-acyl carrier protein synthases I and II of Escherichia coli. J. Biol. Chem. 255, 11949-11956 (1980).

43. Wang, J. et al. Platensimycin is a selective FabF inhibitor with potent antibiotic properties. Nature 441, 358-361 (2006).

44. Olsen, J. G., Kadziola, A., von Wettstein-Knowles, P., Siggaard-Andersen, M. \& Larsen, S. Structures of $\beta$-Ketoacyl-Acyl carrier protein synthase I complexed with fatty acids elucidate its catalytic machinery. Structure $\mathbf{9}$, 233-243 (2001).

45. Zondlo, N. J. Aromatic-proline interactions: electronically tunable $\mathrm{CH} / \pi$ interactions. Acc. Chem. Res. 46, 1039-1049 (2013).

46. Robbins, T., Liu, Y. C., Cane, D. E. \& Khosla, C. Structure and mechanism of assembly line polyketide synthases. Curr. Opin. Struct. Biol. 41, 10-18 (2016).

47. Dreier, J., Shah, A. N. \& Khosla, C. Kinetic analysis of the actinorhodin aromatic polyketide synthase. J. Biol. Chem. 274, 25108-25112 (1999).

48. Keatinge-Clay, A. T., Maltby, D. A., Medzihradszky, K. F., Khosla, C. \& Stroud, R. M. An antibiotic factory caught in action. Nat. Struct. Mol. Biol. 11 888 (2004).

49. Hertweck, C., Luzhetskyy, A., Rebets, Y. \& Bechthold, A. Type II polyketide synthases: gaining a deeper insight into enzymatic teamwork. Nat. Prod. Rep. 24, 162-190 (2007)

50. Du, D., Katsuyama, Y., Shin-ya, K. \& Ohnishi, Y. Reconstitution of a Type II polyketide synthase that catalyzes polyene formation. Angew. Chem. Int. Ed. 57, 1954-1957 (2018).

51. Du, D. et al. Production of a novel amide-containing polyene by activating a cryptic biosynthetic gene cluster in Streptomyces sp. MSC090213JE08. ChemBioChem 17, 1464-1471 (2016).

52. Grammbitter, G. L. C. et al. An uncommon type II PKS catalyzes biosynthesis of aryl polyene pigments. J. Am. Chem. Soc. https://doi.org/10.1021/ jacs.8b10776 (2019).

53. Cronan, J. E. The chain-flipping mechanism of ACP (acyl carrier protein)dependent enzymes appears universal. Biochem. J. 460, 157 LP-163 (2014)

54. Pappenberger, G., Schulz-Gasch, T., Kusznir, E., Müller, F. \& Hennig, M. Structure-assisted discovery of an aminothiazole derivative as a lead molecule for inhibition of bacterial fatty-acid synthesis. Acta Crystallogr. Sect. D. 63 , 1208-1216 (2007)

55. Henzler-Wildman, K. \& Kern, D. Dynamic personalities of proteins. Nature 450, 964 (2007)

56. Traitenberg, F. et al. Structural insights into bacterial resistance to cerulenin Febs J. 281, 2324-2338 (2014)

57. Robbins, T., Kapilivsky, J., Cane, D. E. \& Khosla, C. Roles of conserved active site residues in the ketosynthase domain of an assembly line polyketide synthase. Biochemistry 55, 4476-4484 (2016).

58. Kosa, N. M., Haushalter, R. W., Smith, A. R. \& Burkart, M. D. Reversible labeling of native and fusion-protein motifs. Nat. Meth 9, 981-984 (2012).

59. Liu, H. \& Naismith, J. H. An efficient one-step site-directed deletion, insertion, single and multiple-site plasmid mutagenesis protocol. BMC Biotechnol. 8, 91 (2008).

60. Schneider, C. A., Rasband, W. S. \& Eliceiri, K. W. NIH Image to ImageJ: 25 years of image analysis. Nat. Methods 9, 671-675 (2012).

61. Battye, T. G. G., Kontogiannis, L., Johnson, O., Powell, H. R. \& Leslie, A. G. W. iMOSFLM: a new graphical interface for diffraction-image processing with MOSFLM. Acta Crystallogr. D. Biol. Crystallogr. 67, 271-281 (2011).

62. Winn, M. D. et al. Overview of the CCP4 suite and current developments. Acta Crystallogr. D. Biol. Crystallogr. 67, 235-242 (2011).

63. Lebedev, A. A. et al. JLigand: a graphical tool for the CCP4 template-restraint library. Acta Crystallogr. D. Biol. Crystallogr. 68, 431-440 (2012). 
64. Adams, P. D. et al. PHENIX: a comprehensive python-based system for macromolecular structure solution. Acta Crystallogr. D. Biol. Crystallogr. 66, 213-221 (2010).

65. Roujeinikova, A. et al. Structural studies of fatty Acyl-(Acyl Carrier Protein) thioesters reveal a hydrophobic binding cavity that can expand to fit longer substrates. J. Mol. Biol. 365, 135-145 (2007).

66. Hanwell, M. D. et al. Avogadro: an advanced semantic chemical editor, visualization, and analysis platform. J. Cheminform. 4, 1 (2012).

67. Gordon, J. C. et al. $\mathrm{H}++$ : a server for estimating pKas and adding missing hydrogens to macromolecules. Nucleic Acids Res. 33, W368-71 (2005).

68. Myers, J., Grothaus, G., Narayanan, S. \& Onufriev, A. A simple clustering algorithm can be accurate enough for use in calculations of $\mathrm{pKs}$ in macromolecules. Proteins 63, 928-938 (2006).

69. Anandakrishnan, R., Aguilar, B. \& Onufriev, A. V. H++3.0: automating pK prediction and the preparation of biomolecular structures for atomistic molecular modeling and simulations. Nucleic Acids Res. 40, W537-W541 (2012).

70. Bashford, D. \& Karplus, M. pKa's of ionizable groups in proteins: atomic detail from a continuum electrostatic model. Biochemistry 29, 10219-10225 (2002).

71. Maier, J. A. et al. ff14SB: improving the accuracy of protein side chain and backbone parameters from ff99SB. J. Chem. Theory Comput. 11, 3696-3713 (2015).

72. Wang, J., Wolf, R. M., Caldwell, J. W., Kollman, P. A. \& Case, D. A. Development and testing of a general AMBER force field. J. Comput. Chem. 25, 1157-1174 (2004).

73. Wang, J., Wang, W., Kollman, P. A. \& Case, D. A. Automatic atom type and bond type perception in molecular mechanical calculations. J. Mol. Graph. Model. 25, 247260 (2006).

74. Bayly, C. I., Cieplak, P., Cornell, W. \& Kollman, P. A. A well-behaved electrostatic potential based method using charge restraints for deriving atomic charges: the RESP model. J. Phys. Chem. 97, 10269-10280 (2002).

75. Jorgensen, W. L., Chandrasekhar, J., Madura, J. D., Impey, R. W. \& Klein, M. L. Comparison of simple potential functions for simulating liquid water. $J$. Chem. Phys. 79, 926-935 (1998)

76. Case, D.A. et al., AMBER 2016 (University of California, San Francisco, 2016).

77. Ryckaert, J.-P., Ciccotti, G. \& Berendsen, H. J. C. Numerical integration of the cartesian equations of motion of a system with constraints: molecular dynamics of n-alkanes. J. Comput. Phys. 23, 327-341 (1977).

78. Darden, T., York, D. \& Pedersen, L. Particle mesh Ewald: An N. $\log (\mathrm{N})$ method for Ewald sums in large systems. J. Chem. Phys. 98, 10089-10092 (1998).

79. Liu, J., Li, D. \& Liu, X. A simple and accurate algorithm for path integral molecular dynamics with the Langevin thermostat. J. Chem. Phys. 145, 024103 (2016).

80. Roe, D. R., Thomas, E. \& Cheatham, I. PTRAJ and CPPTRAJ: software for processing and analysis of molecular dynamics trajectory data. J. Chem. Theory Comput. 9, 3084-3095 (2013).

81. McGibbon, R. T. et al. MDTraj: a modern open library for the analysis of molecular dynamics trajectories. Biophys. J. 109, 1528-1532 (2015).

82. Humphrey, W., Dalke, A. \& Schulten, K. VMD—visual molecular dynamics. J. Molec. Graph. 14, 33-38 (1996).

\section{Acknowledgements}

This research was supported by National Science Foundation Grant EEC-0813570 (to J.P.N.) and NIH GM095970 (to M.D.B.). NIH grant T32 GM008326 for support of J.T.M. NIH
Grant T32 GM112584 for support of T.G.B. T.D.D. is a San Diego IRACDA Postdoctoral Fellow supported by NIH K12 GM068524. Portions of the work were also funded by the Arthur and Julie Woodrow Chair at the Salk Institute (to J.P.N.) and the Howard Hughes Medical Institute (to J.P.N.). The authors thank Dr Gordon Louie for assistance in X-ray data collection, processing, and refinement and Marianne Bowman for assistance with protein crystallization. The authors also thank Dr Laetitia Misson for assistance with data analysis. We further acknowledge the following organizations for support of the computer simulation work reported herein: NIH GM31749 (to J.A.M.) and the San Diego Supercomputing Center (to J.A.M.).

\section{Author contributions}

J.J.L., J.A.M, M.D.B., and J.P.N. designed and supervised the project. T.D.D. synthesized probe molecules. J.T.M, W.E.K., T.G.B., and A.C. purified protein, optimized crosslinking, carried out crystallization, and obtained crystals. J.T.M. collected x-ray diffraction data and refined and analyzed crystallographic models. J.T.M designed and produced gating mutants. W.E.K. and A.C. performed crosslinking assays. A.P. performed MD simulations. A.P., J.T.M., and J.A.M analyzed simulation data. J.T.M., A.P., J.J.L., J.A.M., M.D.B., and J.P.N. wrote the manuscript. All authors edited the manuscript. W.E.K., T.D.D., A.C., and T.G.B. contributed equally to this work.

\section{Competing interests}

The authors declare no competing interests.

\section{Additional information}

Supplementary information is available for this paper at https://doi.org/10.1038/s41467020-15455-x.

Correspondence and requests for materials should be addressed to J.P.N. or M.D.B.

Peer review information Nature Communications thanks Martin Grininger, and the other, anonymous, reviewer(s) for their contribution to the peer review of this work. Peer reviewer reports are available.

Reprints and permission information is available at http://www.nature.com/reprints

Publisher's note Springer Nature remains neutral with regard to jurisdictional claims in published maps and institutional affiliations.

Open Access This article is licensed under a Creative Commons Attribution 4.0 International License, which permits use, sharing, adaptation, distribution and reproduction in any medium or format, as long as you give appropriate credit to the original author(s) and the source, provide a link to the Creative Commons license, and indicate if changes were made. The images or other third party material in this article are included in the article's Creative Commons license, unless indicated otherwise in a credit line to the material. If material is not included in the article's Creative Commons license and your intended use is not permitted by statutory regulation or exceeds the permitted use, you will need to obtain permission directly from the copyright holder. To view a copy of this license, visit http://creativecommons.org/ licenses/by/4.0/

(C) The Author(s) 2020 